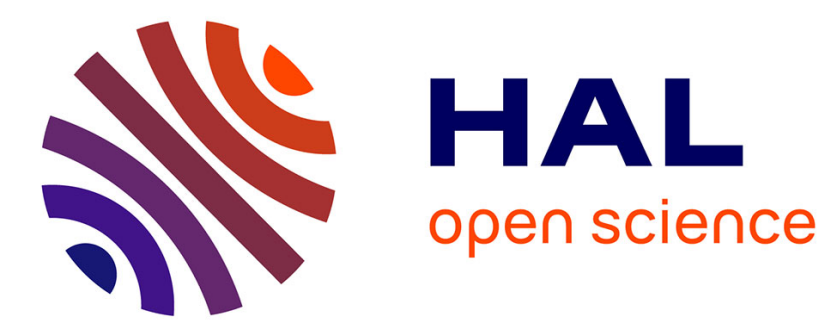

\title{
Firm heterogeneity and the pattern of R\&D collaborations
}

Pascal Billand, Christophe Bravard, Jacques Durieu, Sudipta Sarangi

\section{To cite this version:}

Pascal Billand, Christophe Bravard, Jacques Durieu, Sudipta Sarangi. Firm heterogeneity and the pattern of R\&D collaborations. 2018. hal-01905345

\section{HAL Id: hal-01905345 \\ https://hal.univ-grenoble-alpes.fr/hal-01905345}

Preprint submitted on 25 Oct 2018

HAL is a multi-disciplinary open access archive for the deposit and dissemination of scientific research documents, whether they are published or not. The documents may come from teaching and research institutions in France or abroad, or from public or private research centers.
L'archive ouverte pluridisciplinaire HAL, est destinée au dépôt et à la diffusion de documents scientifiques de niveau recherche, publiés ou non, émanant des établissements d'enseignement et de recherche français ou étrangers, des laboratoires publics ou privés. 


\section{MPRA \\ Munich Personal RePEc Archive}

\section{Firm Heterogeneity and the Pattern of R\&D Collaborations}

Pascal Billand and Christophe Bravard and Jacques Durieu and Sudipta Sarangi

Virginia Tech

August 2018

Online at https://mpra.ub.uni-muenchen.de/89247/

MPRA Paper No. 89247, posted 18 October 2018 13:37 UTC 


\title{
Firm Heterogeneity and the Pattern of R\&D Collaborations ${ }^{1}$
}

\author{
Pascal Billand $^{a}$, Christophe Bravard ${ }^{b}$, Jacques Durieu $^{b}$, Sudipta Sarangi ${ }^{c}$ \\ ${ }^{a}$ Université de Lyon, Lyon, F-69003, France ; Université Jean Monnet, Saint-Etienne, F-42000, France; \\ CNRS, GATE Lyon St Etienne, Saint-Etienne, F-42000, France; pascal.billand@univ-st-etienne.fr \\ ${ }^{b}$ Université Grenoble-Alpes, GAEL, GATE Lyon St Etienne; christophe.bravard@univ-grenoble-alpes.fr, \\ jacques.durieu@univ-grenoble-alpes.fr \\ ${ }^{c}$ DIW Berlin and Department of Economics, Virginia Tech Blacksburg VA 24061 - 0316, USA; \\ ssarangi@vt.edu.
}

August 2018

\begin{abstract}
We consider an oligopoly setting in which firms form pairwise collaborative links in R\&D with other firms. Each collaboration generates a value that depends on the identity of the firms that collaborate. First, we provide properties satisfied by pairwise equilibrium networks and efficient networks. Second, we use these properties in two types of situation (1) there are two groups of firms, and the value of a collaboration is higher when firms belong to the same group; (2) some firms have more innovative capabilities than others. These two situations provide clear insights about how firms heterogeneity affects both equilibrium and efficient networks. We also show that the most valuable collaborative links do not always appear in equilibrium, and a public policy that increases the value of the most valuable links may lead to a loss of social welfare.
\end{abstract}

JEL classification: C70, L13, L20;

Key Words: Networks, R\&D collaborations, link value heterogeneity.

\footnotetext{
${ }^{1}$ We thank participants at Auburn University, Virginia Tech, the Association for Public Economic Theory, Luxemburg, 2015; Royal Economic Society Meetings, 2015; 10th Annual Conference on Economic Growth and Development, ISI Delhi, 2014. P. Billand, C. Bravard and J. Durieu are supported by the grant ANR Renewal.
} 


\section{Introduction}

R\&D collaboration among firms is now quite widespread, especially in industries characterized by rapid technological change like pharmaceutical, chemical and IT industries. Interestingly vast majority of these collaborations are bilateral (see for instance Hagedoorn, 2002), prompting questions about the structural features of the network of R\&D collaborations and their impact on industry performance (Powell et al. 2005). The architecture of these networks is typically asymmetric. Moreover, it is often possible to find the simultaneous co-existence of firms having intense collaborations with other firms engaging minimally in collaborative activities (Powell et al. 2005). Another key feature that comes across from the empirical literature is that the identities of the collaborating firms is important for understanding the resulting networks (see for instance Vonrotas and Okamura, 2009, Vonortas, 2015).

Keeping these stylized facts in mind, our paper develops a model of R\&D collaboration among horizontally related firms where the outcome of the collaboration depends on the characteristics or identity of the firm (as shown in Mowery et al. 1998 and Gomes-Casseres, Hagendorn and Jaffe, 2006). The paper incorporates an interesting trade-off: while interfirm collaboration lowers costs of production, it also serves to increase competition among rival firms. Following Goyal and Joshi (2003; henceforth GJ), we set up a two oligopoly stage model where firm establish their collaborative links in stage 1 and compete in stage 2. The pairwise collaborative links for R\&D purposes in our model require a commitment of resources on the part of the collaborating firms, viewed as the costs of link formation, and lead to lower production costs. The cost-reducing impact of collaborations is link-specific to capture the identity of the firms in question. ${ }^{2}$

We begin by examining the classic homogeneous product model of quantity competition and identify properties of the equilibrium and efficient networks for this case. This helps to establish results for our main question of interest: what is the architecture of stable and efficient networks?

\footnotetext{
${ }^{2}$ This value may depend for instance on the geographic or technological proximity of firms.
} 
Given that our model allows for link specific heterogeneity, a systematic technique is needed to obtain insights about the pattern of collaborations that will occur in equilibrium networks and welfare-maximizing networks. We do this by introducing two stylized frameworks where we limit the heterogeneity in the cost-reduction parameter in a systematic way. These frameworks allow to take into consideration two types of heterogeneity that are often cited as playing an important role in the choice of collaborative partners and the result of collaborations (see among others Mowery et al., 1998, Gomes-Casseres, et al., 2006, Vonortas and Okamura, 2009, Vonortas and Zirulia, 2015, Blum et al., 2017).

(1) In the Insider-Outsider framework (I-O framework), we assume that firms belong to two distinct groups and the value of a collaboration between two firms is higher if these firms belong to the same group than if they belong to different groups. This allows us to have two possible levels of cost-reduction. Such an heterogeneity highlights the role of different kinds of proximities (e.g., technical, geographical, ...) between firms in the choice and the value of interfirm collaboration.

(2) In the High and Low innovative firms framework (H-L framework), we assume that firms either have high or low innovative potential. The value of collaboration between high innovative potential firms is higher than the corresponding value between two low innovative potential firms. The cost of production when a firm with innovative potential collaborates with a firm having low innovative potential lies in between the other two costs. Thus in this framework, we have three different cost reduction parameters. This framework relies on the well-established facts that firms differ in their posture toward innovation and that firms with a more dynamic posture toward innovation are more attractive as collaborative partners. ${ }^{3}$

Architectures that arise in equilibrium networks in these two frameworks are either of a groupdominant type or of a hierarchical type. ${ }^{4}$ We then identify the architectures of efficient networks

\footnotetext{
${ }^{3}$ The I-O framework can be seen as an horizontal heterogeneity and the H-L framework as a vertical heterogeneity. We thank one of the referees for this distinction.

${ }^{4}$ In a group-dominant type architecture, there are sets of firms such that firms belonging to a set are linked with all firms in this set, but have no other links. In a hierarchical type architecture, there is a set of firms $N^{\prime}$ such that for every firm $i$ that has formed a link, there exists a firm $j \in N^{\prime}$ that is linked with $i$.
} 
in the I-O and H-L frameworks. ${ }^{5}$ We establish that in both frameworks efficient networks are of a nested split graph/network type. ${ }^{6}$ Finally, we go beyond the linear oligopoly model and demonstrate how our results can be generalized to a larger class of games that includes both the differentiated Cournot and Bertrand oligopoly models.

Our paper is a contribution to the study of network formation and cooperation in oligopolies. The model of collaborative networks we present is inspired by recent research on R\&D networks and is most directly related to GJ (2003). The authors assume that collaborative R\&D links are homogeneous, that is they have the same value in terms of their cost-reducing impact. Our model depart from this assumption, and considers by allowing for the value of the cost-reducing parameter to vary across links, and provides a systematic way to obtain insights under links cost heterogeneity. Additionally, since our model incorporates the identity of the collaborating firms, we find that the pattern of equilibrium networks is asymmetric, which is closer to reality. In the GJ model, in equilibrium, either all firms are similar (form a complete network or an empty network), or there are two groups of firms (in the group-dominant network). However, in the latter case, firms belonging to a group have the same number of links, therefore have the same marginal cost and competitiveness in the market. The introduction of link-specific heterogeneity in our paper implies a much greater variability in the degree of competitiveness among firms. In particular, with just two different sets of link values, hierarchical architectures already emerge in equilibrium.

Finally, our model also allows us to give some interesting insights over public policy. In the paper, we find that the most valuable links are not always formed in equilibrium networks. This implies that more profitable innovations do not always occur in equilibrium. However we show that public intervention aimed at promoting the most valuable links can be counterproductive.

Our paper also relates to Westbrock (2010) who provides architectures of efficient networks for

\footnotetext{
${ }^{5}$ Note that while we only consider two types of firms in the I-O and H-L frameworks, our results are qualitatively preserved when we allow for a greater number of firm types.

${ }^{6}$ Roughly speaking in a nested split graph, the neighborhoods of the firms are nested together.
} 
homogeneous link values. Billand et al. (2015) refine his result and show that efficient networks are nested split graphs using conditions on individual firm payoffs rather than aggregate payoffs. ${ }^{7}$ Our paper finds the conditions on individual payoffs when link values are heterogeneous.

We now briefly point out some other papers to which our work relates as well. Goyal and Moraga-Gonzales (2001), Goyal, Moraga-Gonzales, and Konovalov (2008) analyze the interaction between the effort of firms on collaborative links and the effort of firms on other R\&D projects, under link cost heterogeneity. Our paper also complements the work of König et al. (2012) who examine stability and efficiency of R\&D networks in a model with network dependent spillovers. The presence of both direct and indirect spillovers in the analysis of König et al. is an important feature of their model that distinguishes it from the GJ's framework. Not surprisingly, in their paper, because of the benefits from indirect links, every firm in a connected component has the same profit.

The rest of the paper is organized as follows. In section 2, we present the model setup. In section 3 , we provide the results for a market with homogeneous products under quantity competition. In section 4 , we propose a generalized framework which allows us to deal with differentiated oligopolies. In section 5, we conclude and also discuss the differences between our framework and heterogeneous link formation costs.

\section{Model setup}

Network definitions. We consider an industry with a set $N=\{1, \ldots, n\}$ of firms. In the game we model, every firm first announces its intended R\&D collaboration links: $s_{i, j}=1$ means that firm $i$ intends to form a collaborative link with firm $j$, and $s_{i, j}=0$ otherwise. Firms only play pure strategies. Thus a strategy of firm $i$ is given by $s_{i}=\left\{\left\{s_{i, j}\right\}_{j \in N \backslash\{i\}}\right\}$. Let $S_{i}$ denote the strategy set

\footnotetext{
${ }^{7}$ For more on the properties of nested split graphs, see the recent paper by König et al. (2014).
} 
of firm $i$. The set $S=\times_{j \in N} S_{j}$ is the set of strategy profiles of firms. A link $i j$ between two firms $i$ and $j$ is formed if and only if $s_{i, j}=s_{j, i}=1$. A strategy profile $s=\left\{s_{1}, s_{2}, \ldots, s_{n}\right\}$ therefore induces a network $g^{[s]}$. For expositional simplicity we will often omit the dependence of the network on the underlying strategy profile. A network $g$ is a formal description of the pairwise links, representing R\&D collaboration, that exist between the firms. If firms $i$ and $j$ are linked in $g$, we say $i j \in g$, and we say $i j \notin g$ otherwise. The number of links in $g$ is denoted by $|g|$.

Let $g(i)$ be the set of firms with whom $i$ has formed a link. The cardinality of $g(i)$, denoted by $|g(i)|$, is called the degree of firm $i$. We denote by $\mathcal{N}(g)$ the set of firms that are involved in at least one link in $g$. We set $g_{-i}$ as the network formed when firm $i$ and its links are deleted, $g+i j$ as the network obtained when the link $i j$ is added to $g$, and $g-i j$ as the network obtained when the link $i j$ is removed from $g$.

Network architectures. The empty network is the network with no links. The complete network is the network where every firm $i$ is linked with each other firm. In the paper, we also find other equilibrium network architectures, some of them are illustrated in Figure 1. In the figure, every circle represents a set of firms who have the same neighbors; these firms have formed the same number of links. If a circle is shaded in white, then firms belonging to the set associated to this circle are all linked together, while there are no links between these firms when the circle is shaded in grey. Moreover, a line between two sets of firms, say $\mathcal{N}_{1}$ and $\mathcal{N}_{2}$, indicates that there is a link between any two firms $i \in \mathcal{N}_{1}$ and $j \in \mathcal{N}_{2}$.

In a group-dominant network, there is a set of firms that are all linked together and there are no other links. In a 2-group-dominant network, there is a partition of $\mathcal{N}(g)$ in two sets, such that firms that belong to a set are linked together, but there are no other links.

In a 2|2-hierarchical network there is a partition of $\mathcal{N}(g),\left\{\mathcal{N}_{1}, \mathcal{N}_{2}, \mathcal{N}_{3}, \mathcal{N}_{4}\right\}$, such that firms that belong to a set are all linked together, and firms in $\mathcal{N}_{1}$ and $\mathcal{N}_{2}$ are linked together. Moreover, each firm $i \in \mathcal{N}_{1}$ is linked with every firm $j \in \mathcal{N}_{3}$ and each firm $i \in \mathcal{N}_{2}$ is linked with every firm 
$j \in \mathcal{N}_{4}$, and there are no other links. Firms in $\mathcal{N}_{1}$ and $\mathcal{N}_{2}$ are called influential firms, and firms in $\mathcal{N}_{3}$ and $\mathcal{N}_{4}$ are called influenced firms. In a 1|2-hierarchical network there is a partition of $\mathcal{N}(g)$, $\left\{\mathcal{N}_{1}, \mathcal{N}_{2}, \mathcal{N}_{3}\right\}$, such that firms that belong to a set are all linked together, each firm $i \in \mathcal{N}_{1}$ is linked with every firm in $j \in \mathcal{N}_{2} \cup \mathcal{N}_{3}$ and there are no other links. Firms that belong to $\mathcal{N}_{1}$ are called influential firms. A 1|(1,1)-hierarchical network is similar to a 1|2-hierarchical network except that there are no links between firms in $\mathcal{N}_{3}$.

In the paper, we find that efficient networks are nested split graph (NSG) types. Basically, in a NSG, the neighborhood of a node is contained in the neighborhoods of the nodes with higher degrees. ${ }^{8}$ The nested property implies that there is a partition of firms $\left(D_{0}, D_{1}, \ldots, D_{m}\right)$ such that firms which belong to $D_{\ell}, \ell \in\{0, \ldots, m-1\}$, have a lower degree that firms which belong to $D_{\ell+1}$. In a $2-N S G$ there is a partition of $\mathcal{N}(g),\left\{\mathcal{N}_{1}, \mathcal{N}_{2}\right\}$, such that firms in each set form a NSG. The NSG property implies that there is a partition of firms in group $k, k=1,2,\left(D_{0}^{k}, D_{1}^{k}, \ldots, D_{m}^{k}\right)$ such that firms which belong to $D_{\ell}^{k}$ have a lower degree that firms which belong to $D_{\ell+1}^{k}$. A multi-NSG is a 2-NSG, with firms in $\mathcal{N}_{1}$ and firms in $\mathcal{N}_{2}$ forming a NSG, and each firm $i \in D_{4}^{1}\left(D_{4}^{2}\right)$ is linked with all firms $i^{\prime}$ that are linked with a firm $j \in D_{3}^{1}\left(D_{3}^{2}\right)$. A group-NSG is similar to a multi-NSG except that there are no links between firms in $\mathcal{N}_{2}$.

Values of links. To capture heterogeneity inherent in $R \& D$ collaboration, we assume that each $R \& D$ collaboration is valued differently by the firms. Since $R \& D$ collaborations (viewed as link) reduce costs for the firm, we assume that different $R \& D$ collaborations can reduce costs to different extents. This captures the idea that the innovation targeted by one link is different from the innovation targeted by another link. Let $v_{i, j}>0$ be the exogenously given value of the links $i j$ for the firm $i$. We assume that a link between two collaborating firms $i$ and $j$ has the same value for the two firms involved, that is $v_{i, j}=v_{j, i}$, and we set $\bar{v}=\max \left\{v_{i, j}\right\}$. In the following, we say that link $i j$ is more valuable than link $i^{\prime} j^{\prime}$ if $v_{i, j}>v_{i^{\prime}, j^{\prime}}$.

\footnotetext{
${ }^{8}$ In Appendix A, we formally define NSG.
} 


$$
\begin{array}{lll}
1-2 & 5 & 6 \\
|\times| & & \\
3-4 & 7 & 8
\end{array}
$$

A group-dominant network

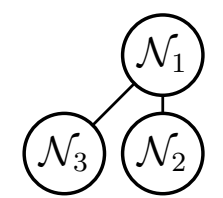

A 1|2-hierarchical network

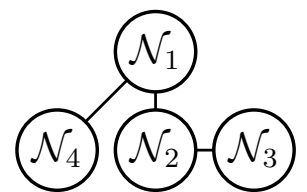

A 2|2-hierarchical network

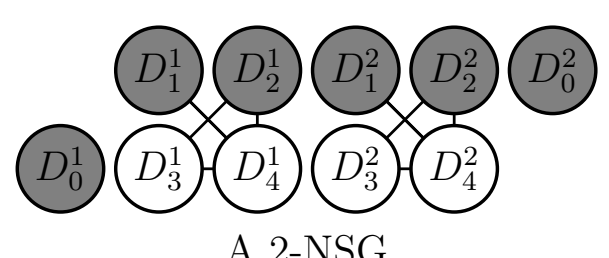

$$
\begin{array}{ll}
1-2 & 5-6 \\
|\times| & \mid / 8 \\
3-4 & 7
\end{array}
$$

A 2-group-dominant network

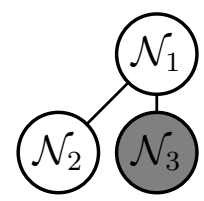

A 1|(1,1)-hierarchical network

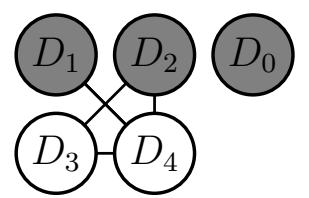

A NSG

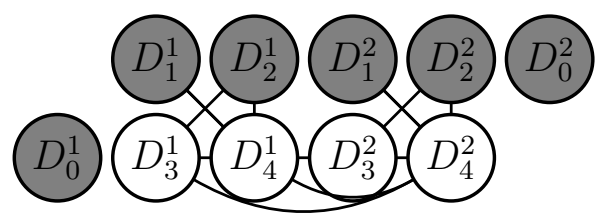

A multi-NSG

Figure 1: Specific network architectures 
We associate with each firm $i$ a number $\mathcal{V}_{i}(g)=\sum_{i j \in g(i)} v_{i, j}$ representing its "flow degree". We set $\mathcal{V}(g)=\sum_{i j \in g, i>j} v_{i, j}$ the sum of the flow degree of firms, and $\mathcal{V}\left(g_{-i}\right)=\mathcal{V}(g)-\mathcal{V}_{i}(g)$ the sum of the flow degree of firms in network $g_{-i}$.

Structure of the game. The game played by the firms consists of two stages.

1. Stage 1: Firms simultaneously choose the collaborative links they intend to form in order to decrease their marginal cost.

2. Stage 2: Firms play a simultaneous oligopoly game, given the network formed in the first stage.

Pairwise equilibrium network. We use the notion of pairwise equilibrium network defined by Goyal and Joshi (2006) to characterize the architectures of the networks formed by profit maximizing firms.

First, we define a Nash equilibrium. Let $S_{-i}=\times_{j \in N \backslash\{i\}} S_{j}$ be the joint strategy set of all firms except $i$, with $s_{-i}$ a typical member of $S_{-i}$, and let $\pi_{i}^{\star}\left(g^{\left[s_{i}, s_{-i}\right]}\right)$ be the oligopoly equilibrium profit of firm $i$ in the second stage, given the strategy profile $s=\left(s_{i}, s_{-i}\right)$ played by the firms in the first stage. The strategy $s_{i} \in S_{i}$ is said to be a best response of firm $i$ to $s_{-i} \in S_{-i}$ if $\pi_{i}^{\star}\left(g^{\left[s_{i}, s_{-i}\right]}\right) \geq \pi_{i}^{\star}\left(g^{\left[s_{i}^{\prime}, s_{-i}\right]}\right)$, for all $s_{i}^{\prime} \in S_{i}$. The set of firm $i^{\prime} s$ best responses to $s_{-i}$ is denoted by $\operatorname{BR}_{i}\left(s_{-i}\right)$. A strategy profile $s \in S$ is said to be a Nash equilibrium if $s_{i} \in \mathrm{BR}_{i}\left(s_{-i}\right)$, for all $i \in N$. In the following, to simplify notation we replace $\pi_{i}^{\star}\left(g^{[s]}\right)$ by $\pi_{i}^{\star}(g)$.

Definition 1 (Goyal and Joshi, p. 324, 2006) A network $g$ is a pairwise equilibrium network if the following conditions hold:

1. There is a Nash equilibrium strategy profile which supports $g$.

2. For $g_{i, j}=0, \pi_{i}^{\star}(g+i j)-\pi_{i}^{\star}(g)>0 \Rightarrow \pi_{j}^{\star}(g+i j)-\pi_{j}^{\star}(g)<0$. 
Observe that a pairwise equilibrium network is a refinement of Nash equilibrium: it is a Nash equilibrium where there does not exist a pair of firms with an incentive to form a link.

Efficient network. We define social welfare as the sum of consumers surplus, CS, and total profit of firms: $W(g)=C S(g)+\sum_{i \in N} \pi_{i}^{\star}(g)$.

Definition 2 An efficient network is a network that maximizes social welfare.

\section{Homogeneous Cournot Game}

In this section, we consider the textbook linear oligopoly model. To simplify the analysis, following much the literature, we assume that the marginal cost function of a firm decreases linearly with its flow degree:

$$
c_{i}(g)=\gamma_{0}-\gamma \sum_{j \in g(i)} v_{i, j}=\gamma_{0}-\gamma \mathcal{V}_{i}(g),
$$

where $\gamma>0, \gamma_{0}>\gamma(n-1) \bar{v}$. In Equation 1, the flow degree, $\mathcal{V}_{i}(g)$, can be interpreted as the impact of the innovations from the collaborative links in lowering firm $i$ 's marginal costs.

Demand and profit functions. We assume the following linear inverse demand function:

$$
p=\alpha-\sum_{i \in N} q_{i}, \alpha \geq 0
$$

where $p$ is the market price of the good and $q_{i}$ is the quantity sold by firm $i$.

Given any network $g$, the Cournot equilibrium output is:

$$
q_{i}^{\star}(g)=\frac{\alpha-\gamma_{0}+n \gamma \mathcal{V}_{i}(g)-\gamma \sum_{j \in N \backslash\{i\}} \mathcal{V}_{j}(g)}{n+1}
$$


In this section, we assume that we have $\alpha-\gamma_{0}>2 \gamma(n-1)^{2} \bar{v}$ (labeled C1), ensuring strictly positive output for every firm. We can write the Cournot equilibrium output as follows:

$$
q_{i}^{\star}(g)=a+b \mathcal{V}_{i}(g)-c \mathcal{V}\left(g_{-i}\right)
$$

where $a=\left(\alpha-\gamma_{0}\right) /(n+1), b=\gamma(n-1) /(n+1)$, and $c=2 \gamma /(n+1)$.

The second stage Cournot gross profit of firm $i$ is given by:

$$
\Pi_{i}^{\star}(g)=\varphi\left(\mathcal{V}_{i}(g), \mathcal{V}\left(g_{-i}\right)\right)=\left(a+b \mathcal{V}_{i}(g)-c \mathcal{V}\left(g_{-i}\right)\right)^{2}
$$

We assume that a collaborative link requires a fixed investment $f>0$. Thus, the second stage Cournot profit of firm $i$ is given by

$$
\pi_{i}^{\star}(g)=\Pi_{i}^{\star}(g)-|g(i)| f
$$

The consumer surplus is equal to

$$
C S(g)=1 / 2\left(\sum_{i \in N} q_{i}^{\star}\right)^{2}=1 / 2\left(\frac{n\left(\alpha-\gamma_{0}\right)+2 \gamma \mathcal{V}(g)}{n+1}\right)^{2}=\phi(\mathcal{V}(g))
$$

Given the extent of heterogeneity allowed in the model, it should be clear that we have many degrees of freedom which can make it easy to generate many different results. Moreover, this makes it possibly difficult to obtain insights about what drives the structural properties of equilibrium and efficient networks. Hence to obtain systematic insights about what heterogeneity may add to the problem, we introduce two different scenarios based on stylized facts that restrict the set of collaboration values in a meaningful way.

In the first scenario, called the Insider-outsider model with cost reduction (I-O frame- 
work), we assume that there are two groups of firms, $N_{I O}^{1}$ and $N_{I O}^{2}$, and the value of a link between two firms $i$ and $j$ is higher when they belong to the same group. It is useful to define $N_{I O}^{k}(g) \subset N_{I O}^{k}$, $k \in\{1,2\}$, the set of firms in $N_{I O}^{k}$ that have formed links in network $g$. Moreover, we denote by $N_{I O}^{12}(g)$ the set of firms which have formed inter-group links. We set $v_{i, j}=v^{I}$ for all $i, j \in N_{I O}^{k}$, $k \in\{1,2\}$, and $v_{i, j}=v^{O}<v^{I}$ for all $i \in N_{I O}^{k}, j \in N_{I O}^{k^{\prime}}, k \neq k^{\prime}$. The assumption, $v^{O}<v^{I}$ is in touch with empirical studies. For instance, Gomes-Casseres, Hagedoorn and Jaffe (2006) show that technological, geographical and business similarities between partners have a positive impact on the value of collaboration. Likewise, adopting a resource-based view of the firm, Mowery et al. (1998) argue that some level of technological overlap is required to facilitate knowhow exchange and development. Finally, Vonortas and Zirulia (2015), show that pre-existing knowledge in the partner's field of expertise, and cognitive proximity is required for effective communication and ability to learn from a collaboration.

In the second scenario, called the High and low innovative firms model with cost reduction (H-L framework) $)^{9}$, we assume that there are two groups of firms, $N^{H}$ and $N^{L}$, with $\left|N^{H}\right|=\left|N^{L}\right|$. Firms which belong to $N^{H}$ have a higher innovative potential, that is a higher ability to innovate and to exploit innovations. Let $N^{k}(g) \subset N^{k}, k \in\{H, L\}$, be the set of firms in $N^{k}$ that have formed links in network $g$. Moreover, we define $N^{H L}(g)$ as the set of firms in $H$ which have formed links with firms in $L$ in $g$. We set $v_{i, j}=v^{H}$ for all $i, j \in N^{H}, v_{i, j}=v^{L}$ for all $i, j \in N^{L}$, and $v_{i, j}=v^{M}$ for all $i \in N^{k}, j \in N^{k^{\prime}}, k \neq k^{\prime}$, and assume that $v^{H}>v^{M}>v^{L}$. Again this distinction is a reasonable assumption given what we find in reality. For instance, in an early paper Miller and Friesen (1982) argue that some firms are conservative with regard to pursuing innovation (low innovators) while others are more aggressive with regard to innovation (high innovators). Likewise, Blundel, Griffith and van Reenen (1999) using a sample of British firms find that firms with a greater market share are simultaneously the more innovative ones. We assume that the value of

\footnotetext{
${ }^{9}$ High innovative and low innovative potential firms have been introduced in a context of R\&D networks by Persitz (2014). However, the author's model does not consider the second stage of our game: the oligopoly game.
} 
a collaboration is higher between high potential innovative firms. If a high and low innovative potential collaborate, then we assume that the value of the links lies between the two ranges.

\subsection{Pairwise Equilibrium Networks}

We begin our analysis with the characterization of equilibria. All the proofs are given in Appendix. The first proposition provides some conditions satisfied by pairwise equilibrium networks without any restrictions on the heterogeneity of link value. This proposition is divided into two parts. First, we provide a condition that leads firm $i$ to have formed a link, with value $v_{i, j}$, when it has also formed a link with a value $v$. This condition is based on the comparison between

- the ratio of the values of links $v$ and $v_{i, j}$,

- and the ratio of the average slope of the gross profit function $\varphi$ over $\left[\mathcal{V}_{i}(g), \mathcal{V}_{i}(g)+v_{i, j}\right]$ and over $\left[\mathcal{V}_{i}(g)-v, \mathcal{V}_{i}(g)\right]$.

Second, we provide a condition that leads firm $i^{\prime}$ to have formed a link, with value $v_{i^{\prime}, j^{\prime}}$, when another firm has formed a link with value $v$. This condition is based on the comparison between the average slope of $\varphi$ over $\left[\mathcal{V}_{i}(g), \mathcal{V}_{i}(g)+v\right]$, when $i$ faces $\mathcal{V}\left(g_{-i}\right)$ links, and the average slope of $\varphi$ over $\left[\mathcal{V}_{i^{\prime}}(g), \mathcal{V}_{i^{\prime}}(g)+v_{i^{\prime}, j^{\prime}}\right]$ when $i^{\prime}$ faces $\mathcal{V}\left(g_{-i^{\prime}}\right)$ links. Moreover, this condition allows us to identify a set of situations, defined as the flow degrees of firm $i^{\prime}$ in $g$ and the values of links, in which firm $i^{\prime}$ has an incentive to form links with such values when she faces $\mathcal{V}\left(g_{-i^{\prime}}\right)$.

In order to present this proposition, we introduce: $(i)$ the set $\left(\mathcal{V}_{i}(g), v ; \mathcal{V}\left(g_{-i}\right)\right)_{\geq}$of pairs $\left(\mathcal{V}_{i}\left(g^{\prime}\right), v^{\prime}\right)$ which provide to firm $i$ a marginal profit higher or equal to the pair $\left(\mathcal{V}_{i}(g), v\right)$, given the sum of flows degree $\mathcal{V}\left(g_{-i}\right) ;{ }^{10}$ and $(i i)$ the average of the derivative of function $\varphi$ at $(x+h, y)$ and $(x, y)$, $\bar{\varphi}_{1}^{\prime}(x, y ; h)=\frac{\varphi_{1}^{\prime}(x+h, y)+\varphi_{1}^{\prime}(x, y)}{2} \cdot{ }^{11}$ Finally, let $\mathcal{M}^{v}(g)=\left\{i \in N:\right.$ there exists $i j \in g$ such that $v_{i, j}=$

\footnotetext{
${ }^{10}$ i.e. $\left(\mathcal{V}_{i}(g), v ; \mathcal{V}\left(g_{-i}\right)\right)_{\geq}=\left\{(X, x) \in \mathbb{R}_{+}^{2}: \varphi\left(X+x, \mathcal{V}\left(g_{-i}\right)\right)-\varphi\left(X, \mathcal{V}\left(g_{-i}\right)\right) \geq \varphi\left(\mathcal{V}_{i}(g)+v, \mathcal{V}\left(g_{-i}\right)\right)\right.$ $\left.-\varphi\left(\mathcal{V}_{i}(g), \mathcal{V}\left(g_{-i}\right)\right)\right\}$

${ }^{11}$ In the appendix, we establish that in our context, the average slope of $\phi$ over $[x, x+h]$, given $y$, is captured by $\bar{\varphi}_{1}^{\prime}(x, y ; h)$.
} 
$v\}$.

Proposition 1 Let $g$ be a pairwise equilibrium network. We assume that firm $i$ belongs to the set $\mathcal{M}^{v}(g)$ and firm $j$ belongs to the set $\mathcal{M}^{v^{\prime}}(g)$, with $v \geq v^{\prime}$.

1. If

$$
\frac{v}{v_{i, j}} \leq \frac{\bar{\varphi}_{1}^{\prime}\left(\mathcal{V}_{i}(g), \mathcal{V}\left(g_{-i}\right) ; v_{i, j}\right)}{\bar{\varphi}_{1}^{\prime}\left(\mathcal{V}_{i}(g)-v, \mathcal{V}\left(g_{-i}\right) ; v\right)}
$$

then there is a link between firms $i$ and $j$ in $g$.

2. Suppose that there is a link between $i$ and $j$ in $g$ with $v_{i, j}=v$, and $\mathcal{V}_{j^{\prime}}(g) \geq \mathcal{V}_{i^{\prime}}(g)$, and $\mathcal{V}_{i}(g)-v=\mathcal{V}$. Moreover, suppose that $\mathcal{V}_{i^{\prime}}(g)=\mathcal{V}$, and $v_{i^{\prime} j^{\prime}}=v^{\prime} \geq v$, and $v_{i^{\prime} j^{\prime}}$ satisfies $\bar{\varphi}_{1}^{\prime}\left(\mathcal{V}_{i^{\prime}}(g), \mathcal{V}\left(g_{-i^{\prime}}\right) ; v^{\prime}\right) \geq \bar{\varphi}_{1}^{\prime}\left(\mathcal{V}_{i^{\prime}}(g), \mathcal{V}\left(g_{-i^{\prime}}\right)-v ; v\right)$. Then $i^{\prime} j^{\prime} \in g$.

Suppose now that $v_{i^{\prime} j^{\prime}} \neq v^{\prime}$, and $\mathcal{V}_{i^{\prime}}(g) \neq \mathcal{V}_{i}(g)-v$. If $\left(\mathcal{V}_{i^{\prime}}(g), v_{i^{\prime}, j^{\prime}}\right) \in\left(\mathcal{V}, v^{\prime} ; \mathcal{V}\left(g_{-i^{\prime}}\right)\right)_{\geq}$, then, $i^{\prime} j^{\prime} \in g$.

Because the ratio of the average of the slopes measures the convexity of $\varphi$, the first part of the proposition states that the more $\varphi$ is convex in the benefit of link formation, the less $v_{i, j}$ can be relatively to $v$ in a pairwise equilibrium network which does not contain a link between $i$ and $j .{ }^{12}$ Consider now network $g$ where $i, i^{\prime}$ and $j$ are not linked, network $g^{\prime}=g+i j, V_{i}(g)=V_{i^{\prime}}(g)$, and $v_{i, j}=v_{i^{\prime}, j}=v$. The second part of the proposition states that if firm $i$ forms a link with firm $j$, then firm $i^{\prime}$ does not always have an incentive to form a link with $j$ in $g^{\prime}$. Let us illustrate this point through an example.

Example 1 We assume that $a=10, b=2 c=1$, and $f=23$. Consider that $g$ is such that $\mathcal{V}_{i}(g)=3, \mathcal{V}_{-i}(g)=4$ and $i$ has formed a link with value 0.5 . If $\mathcal{V}_{i^{\prime}}(g)=2.5$, then firm $i^{\prime}$ has an

\footnotetext{
${ }^{12}$ Note that the convexity in the benefit of link formation is in line with findings in the literature which show that firms with more collaborations are more likely to enter further collaborative agreements (see Novortas and Ziruela, 2015, for instance). Several explanations have been proposed for this phenomenon. For instance, the collaborating process allows firms to learn how to benefit from collaboration and thus collaborating firms have greater incentives to form additional links. Moreover, collaboration with partners is a source of information about new opportunities for alliances and new partners, and is conducive to the formation of new collaborations. Proposition 1 shows that the convexity property can be satisfied even without these factors.
} 
incentive to form a link with $j^{\prime}$ only when $v_{i^{\prime}, j^{\prime}} \geq 0.5217$. It is worth noting that firms $i^{\prime}$ has the same total flow than $i$ when the latter added the link with value 0.5.

We now present a corollary that captures important features of pairwise equilibrium networks. First, if firm $i$ has formed a link with value $v_{i, j}$, then it has an incentive to form a link with a value $v \geq v_{i, j}$. It follows that if a link between two players is at least as valuable than any other link they have formed, then the link between the players must exist in the pairwise equilibrium network. Second, if firm $j$ has formed a link with value $v$, when it has a total flow equal to $\mathcal{V}_{j}(g)$, then $i$ has an incentive to form a link with value $v_{i}>v$ when it has a total flow $\mathcal{V}_{i}(g) \geq \mathcal{V}_{j}(g)$.

Corollary 1 Let $g$ be a pairwise equilibrium network. We assume that firm $i$ belongs to the set $\mathcal{M}^{v}(g)$ and firm $j$ belongs to the set $\mathcal{M}^{v^{\prime}}(g)$, with $v \geq v^{\prime}$.

1. If $v_{i, j} \geq v$, then $i$ and $j$ are linked in $g$.

2. If $\mathcal{V}_{j^{\prime}}(g) \geq \mathcal{V}_{i^{\prime}}(g) \geq \mathcal{V}_{i}(g)$ and $v_{i^{\prime}, j^{\prime}} \geq v$, then $i^{\prime}$ and $j^{\prime}$ are linked in $g$.

It follows from Corollary 1 that if a link between two firms is at least as valuable than any other link they have formed, then the link between these firms must exist in the pairwise equilibrium. We now use the results obtained in Proposition 1 and Corollary 1 to provide the architectures of pairwise equilibrium networks in the I-O and H-L frameworks.

Corollary 2 Suppose that the assumptions of the I-O framework are satisfied. If $g$ is a non-empty pairwise equilibrium network, then it is a group-dominant network, or a 2-group-dominant network, or a 2|2-hierarchical network, or a 1|2-hierarchical network. Moreover,

1. if $g$ is a 2-group-dominant network, then each component consists of firms which belong to the same group $N_{I O}^{k}, k \in\{0,1\}$;

2. if $g$ is a $2 \mid 2$-hierarchical network, then for any $k \in\{1,2\}$, some firms in $N_{I O}^{k}(g)$ have formed links only with all other firms in $N_{I O}^{k}(g)$, and some other firms in $N_{I O}^{k}(g)$ have formed links 
with firms in $N_{I O}^{-k}(g)$, with $-k \in\{1,2\} \backslash\{k\}$;

3. if $g$ is a 1|2-hierarchical network, then firms in $N_{I O}^{k}(g), k \in\{1,2\}$, have formed links with all other firms in $N_{I O}^{k}(g)$. Moreover, some firms in $N_{I O}^{k}(g)$ have formed links with all other firms in $N_{I O}^{-k}(g)$, with $-k \in\{1,2\} \backslash\{k\}$.

Note that in equilibrium, firms in $N_{I O}^{k}, k \in\{1,2\}$, which have formed links with firms in $N \backslash N_{I O}^{k}$ are also linked with all firms in $N_{I O}^{k}$ that have formed links.

Corollary 3 Suppose that the assumptions of the $H$-L framework are satisfied. If $g$ is a non-empty pairwise equilibrium network, then it is a group-dominant network, or a 2-group-dominant network, or a 1|2-hierarchical network, or a 1|(1,1)-hierarchical network. Moreover,

1. if $g$ is a 2-group-dominant network, then each component consists of firms which belong to the same group $N^{k}, k \in\{H, L\}$, and we have $\left|N^{H}(g)\right|<\left|N^{L}(g)\right|$;

2. if $g$ is a 1|2-hierarchical network, then some firms in $N^{H}(g)$ have formed links only with firms in $N^{H}(g)$, and every firm in $N^{L}(g)$ has formed links with all firms in $N^{L}(g)$ and some firms in $N^{H}(g)$. Moreover, we have $\left|N^{H}(g) \backslash N^{H L}(g)\right|<\left|N^{L}(g)\right|$;

3. if $g$ is a $1 \mid(1,1)$-hierarchical network, then every firm in $N^{H}(g)$ has formed links with all other firms that have formed links, and some firms in $N^{L}(g)$ have formed links only with firms in $N^{H}(g)$.

Note that in a 1|2-hierarchical network or a 1|(1,1)-hierarchical network, influential firms belong to $N^{H}$. Moreover, Proposition 1 allows us to provide additional information. For instance, let $g$ be a 2 group-dominant pairwise equilibrium network, where $N^{H}(g)=3, N^{L}(g)=4, a=10, b=3 / 4$, $c=1 / 4, v^{H}=1$ and $v^{L}=1 / 2$. Then, due to point 1 of Proposition 1 , we have $v^{M} \in[0.94,1]$.

Corollaries 2 and 3 show that once we allow for two different values of links, pairwise equilibrium networks will always tend toward group-dominant or hierarchical networks. Note that the group- 
dominant type result is in line with some empirical studies (see Mowery et al., $1998,{ }^{13}$ Vonortas and Okamura, 2009 ${ }^{14}$ ). These studies find that firms similar - in a technological sense or a geographical proximity sense - are more likely partners. As a result, the networks formed by firms will consist of groups of (relatively similar) firms, densely connected internally, with few links between firms belonging to different groups.

There is also empirical evidence for hierarchical structures. Vonortas and Okamura (2013) examine the ICT sector using data from the last two rounds of the European Research Framework Program. They find that there is a small group of hub firms that are crucial for keeping the network together while another group of non-hub firms provides significant networking activity.

We now show by means of an example a interesting aspect of the $\mathrm{H}-\mathrm{L}$ framework: there exist parameter ranges where the most valuable links may not always be formed.

Example 2 Suppose that the assumptions of the H-L framework are satisfied with $N^{L}=\{1, \ldots, 4\}$ and $N^{H}=\{5, \ldots, 8\}, a=10, b=\frac{3}{4}$, and $c=\frac{1}{4}$. Let $v^{L}=2.5, v^{M}=2.7$, and $v^{H}=3$. If $f=35$, then network $g$ drawn in Figure 2 is a pairwise equilibrium network, where the links that can reduce costs the most do not exist in $g$.

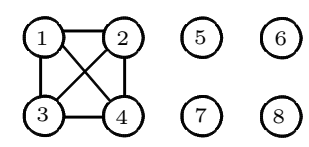

Figure 2: Pairwise equilibrium network without the most valuable links

Given this outcome, one could easily imagine that in order to improve efficiency of the equilibrium, public authorities may device policies that help the firms with the most valuable links to improve the value of these links, say by providing additional resources. However the next example shows that such a policy can lead to an equilibrium where the links that are formed are less valuable

\footnotetext{
${ }^{13}$ The authors use a sample of joint ventures taken from the Cooperative Agreements and Technology Indicators database, a dataset that contains information on over 9000 alliances

${ }^{14}$ The core data for Vonortas and Okamura analysis is drawn from the Innovation Network Databank (INNET).
} 
and, as a result, the social welfare is lower. To simplify the construction of the example, we deal with groups of links instead of groups of firms regarding the differences in the value of links. We assume that because of the public policy, the value of the most valuable links is $k \bar{v}$ instead of $\bar{v}$, with $k>1$.

Example 3 Let $N=\{1, \ldots, 6\}, a=\frac{490}{7}, b=\frac{5}{7}, c=\frac{2}{7}, v^{2}=0.9, v^{1}=0.8918244$, and $f=$ 89.752038. Suppose $v_{i, j}=v^{2}$ for $i j \in\{12,13,45\}, v_{i, j}=v^{1}$ for $i j=23$, and $v_{i, j}=0$ for all other links. Network $g$ in Figure 3 is the unique pairwise equilibrium network. If $k=1.000111$, then, due to the public policy, network $g^{\prime}$ in Figure 3 becomes the unique pairwise equilibrium network. We can check that the total firms' profit and the consumers' surplus are higher in $g^{\prime}$ than in $g$. Thus, in this example, increasing the value of the most valuable links leads to an equilibrium where less valuable links are formed, and social welfare is lower.

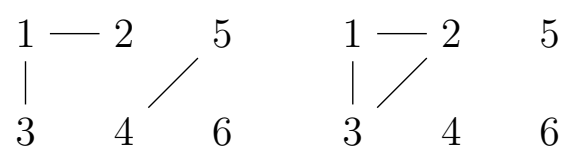

Network $\quad$ Network $g^{\prime}$

Figure 3: Example of non-monotonicity

We now explain the intuition behind Example 3. First, the link 23, which is not the most valuable, is not formed in $g$ but is formed in $g^{\prime}$. This result follows from the convexity of the function $\phi$ with $\mathcal{V}_{i}(g)$ : the increase in the value of the links 12 and 13 makes the formation of the link 23 more attractive for firms 2 and 3. Second, one of most valuable links, the link 45 is formed in $g$ but is not formed in $g^{\prime}$. This results from two opposing forces affecting the incentives to form the link 45: (1) the value of the link 45 is higher, that makes this link more profitable; (2) the number of links formed by firms $j \in N \backslash\{4,5\}$ in $g$ has increased, so forming the link 45 is less profitable for firms 4 and 5. The result in Example 3 follows from the fact that the first force is 
lower than the second one.

Example 3 establishes that there are situations where an increase in the value of the most profitable links by public authorities can be counterproductive since it leads to a decrease in the social welfare. Thus it is important to have a precise idea concerning the properties of networks that maximize the social welfare, and this is done next.

\subsection{Efficient Networks}

We begin with a proposition which establishes that in an efficient network if firm $i$ has formed a link with firm $j$, then firm $i$ has also formed a link with all firms that (a) have a lower marginal cost than $j$, and (b) whose collaboration with $i$ has a higher value than the collaboration between $i$ and $j$.

Proposition 2 Let $g$ be an efficient network that contains a link between firms $i$ and $j$. If $c_{j}(g) \geq$ $c_{j^{\prime}}(g)$ and $v_{i, j} \leq v_{i, j^{\prime}}$, then there is a link between firms $i$ and $j^{\prime}$ in $g$.

The intuition behind Proposition 2 is as follows. First, suppose that firm $j^{\prime}$ has a marginal cost that is lower than $j$ in $g$. Straightforward calculations show that if the link $i j^{\prime}$ has the same value as the link $i j$, then the addition of $i j^{\prime}$ in $g$ implies an increase in welfare that is higher than the increase in welfare associated with the addition of the link $i j$ in $g-i j$. Second, if the link $i j^{\prime}$ has

a higher value than the link $i j$, then it is equivalent to a situation where the link $i j^{\prime}$ has the same value as the link $i j$, and a costless link is added to firms $i$ and $j^{\prime}$. This link allows them to decrease their marginal cost. Since the marginal cost of some firms decrease and the marginal cost of other firms is unchanged, the welfare increases.

We now use Proposition 2 to identify the architectures of efficient networks in different situations. Efficient networks in the framework of cost-reducing collaboration in a homogeneous Cournot game has been studied by Westbrock (Proposition 1, 2010) in situation where links have the same value. His result has been refined by Billand et al. (2015) who show that efficient networks in this 


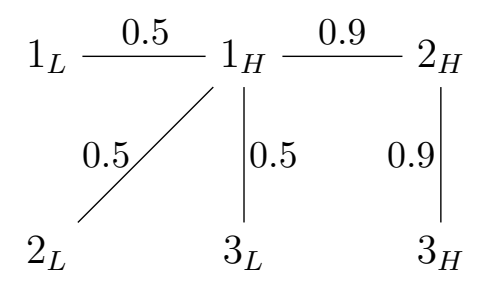

Figure 4: Relationship between flow degree and firms degree in efficient networks

framework are NSG. Here we extend the analysis to the I-O and H-L frameworks where links have different values.

Corollary 4 Suppose that the assumptions of the I-O framework are satisfied. If $g$ is a non-empty efficient network, then it is a NSG, or a 2-NSG, or a group-NSG, or a multi-NSG.

Corollary 5 Suppose that the assumptions of the $H$-L framework are satisfied. If $g$ is a non-empty efficient network, then it is a NSG, or a group-NSG, or a multi-NSG. Moreover, if $g\left[N^{H}\right]=\emptyset$, then $g\left[N^{L}\right]=\emptyset$.

We observe that in both the I-O and H-L frameworks, efficient networks are variations of NSG. We now give the intuition behind this result. Consider Figure 4 in the H-L framework. The flow degree of firm $1_{H}$ is 2.4 and the flow degree of firm $2_{H}$ is 1.8 ; the degree of $1_{H}$ is 4 while the degree of $2_{H}$ is 2. By Proposition 2, network $g$ cannot be an efficient network since firm $1_{H}$ has to add a link with firm $3_{H}$. The resulting network is a NSG which is a candidate to be an efficient network. Since NSG are defined thanks to a property on degrees, this illustrates that the requirement on flow degree for efficiency translates to a requirement on degrees.

The H-L framework provides an easy way to see the possibility of conflict between pairwise equilibrium networks and efficient networks. In particular, in an efficient network, firms in $N^{L}$ will not form links when it is not worthwhile for firms in $N^{H}$ to form links. By contrast, there are pairwise equilibrium where firms in $N^{L}$ form links while firms in $N^{H}$ have no links. 
Moreover, Lemma 1 (in Appendix) provides an interesting result for public authorities in the H-L framework. Indeed, let $g$ be a two-group-dominant pairwise equilibrium network. It results from Lemma 1 that social welfare can be increased if the R \& D collaborative links formed by firm $i \in N^{L}$ with a subset of firms in $A^{L} \subset N^{L}$ in $g$ are replaced by links formed by firm $j \in N^{H}$ with firms in $A^{L} \subset N^{L}$.

Conflict between pairwise equilibrium networks and efficient networks also arises in the I-O framework, and public authorities can act to increase social welfare. For instance, consider a groupdominant network such that the dominant group, as well as the set of isolated firms, contains firms which belong to the two groups. It is easy to show that this network can be a pairwise equilibrium network for some values of the parameters. The following proposition shows how social welfare can be increased in this case. In this proposition, we denote by $C(g)$ the dominant group in the group-dominant network $g$, and by $N_{I O}^{k}(C(g))$ the set of firms in the dominant group $C(g)$ which belong to group $k, k=1,2$.

Proposition 3 Let $g$ be a group-dominant network such that $0<N_{I O}^{1}(C(g))<N_{I O}^{1}$ and $0<$ $N_{I O}^{2}(C(g)) \leq N_{I O}^{2} . \quad$ Wlog suppose $N_{I O}^{1}(g) \geq N_{I O}^{2}(g)$. Suppose $i \in N_{I O}^{1} \backslash N_{I O}^{1}(C(g))$ and $j \in$ $N_{I O}^{2}(C(g))$. Let $g^{\prime}$ be a group-dominant network such that $C\left(g^{\prime}\right)=C(g) \cup\{i\} \backslash\{j\}$. We have $W\left(g^{\prime}\right)>W(g)$.

Moreover, when the size of the two groups are equal, that is $N_{I O}^{1}=N_{I O}^{2}$, we obtain the following corollary.

Corollary 6 Suppose $N_{I O}^{1}=N_{I O}^{2}$. Let g be a group-dominant network such that $0<N_{I O}^{1}(g)<N_{I O}^{1}$ and $0<N_{I O}^{2}(g)<N_{I O}^{2}$. Wlog suppose $N_{I O}^{1}(g) \geq N_{I O}^{2}(g)$. Let $g^{\prime}$ be a a group-dominant network such that $N_{I O}^{1}(g)=N_{I O}^{1}$, and $N_{I O}^{2}(g)=\emptyset$. Then, we have $W\left(g^{\prime}\right)>W(g)$. 


\section{Results for a Larger Class of Oligopoly Games}

In this section, we establish results for cases where the profit function satisfies some general properties. These properties are satisfied not only by our previous model, but also by models of costreducing collaboration in differentiated (Cournot and Bertrand) oligopolies. In the following, we assume that the gross profit function of firm $i$ depends on its flow degree and the flow degree generated by the links in which firm $i$ is not involved:

$$
\Pi_{i}(g)=\sigma\left(\mathcal{V}_{i}(g), \mathcal{V}\left(g_{-i}\right)\right)
$$

We assume that the profit function of firm $i$ is $\pi_{i}(g)=\Pi_{i}(g)-|g(i)| f$, where $f>0$ is the cost of link formation.

Broadly speaking, for a given value, $v$, two types of externality effects arise in our context: an externality across flow degree generated by the links in which firm $i$ is involved, and an externality across flow degree generated by the links in which firm $i$ is not involved. This motivates the following definitions.

Definition 3 The profit function, $\sigma$, is strictly convex in its first argument if for all $y, \sigma(x+v, y)-$ $\sigma(x, y)>\sigma(x, y)-\sigma(x-v, y)$ for all $x$ and $v$ such that $x \geq v>0$.

This definition means that for a given value of links, the higher the flow degree generated by the links in which firm $i$ is involved, the higher is the incentive of firm $i$ to form an additional link. The next definition captures externality across flow degree of different firms.

Definition 4 The gross profit function, $\sigma$, is strictly sub-modular if for all $x>v$, and for all $y^{\prime}>y$ $\sigma\left(x+v, y^{\prime}\right)-\sigma\left(x, y^{\prime}\right)<\sigma(x+v, y)-\sigma(x, y)$.

This definition means that for a given value of links, the higher the flow degree generated by the links in which other firms are involved, the higher is the incentive of firm $i$ to form an additional 
link.

First, we state a proposition that provides necessary conditions for a pairwise equilibrium network. This result complements the work of Goyal and Joshi (Proposition 3.1, p. 327, 2006), when we allow heterogeneity in the values of the links. It is worth noting that our result highlights the fact that a pairwise equilibrium network does not always contain the most valuable links.

Proposition 4 Suppose that the gross payoff function is given by (4) where $\sigma$ is strictly increasing and strictly convex in its first argument, and sub-modular. Let $g$ be a pairwise equilibrium network, with $i j \in g$ and $i j^{\prime} \notin g$. Then, $\mathcal{V}_{j}(g)>\mathcal{V}_{j^{\prime}}(g)$ or $v_{i, j}>v_{i, j^{\prime}}$.

Proposition 4 states that it is not possible to have a situation where simultaneously $(a)$ two unlinked firms $i^{\prime}, j^{\prime}$ have a cost competitive advantage, and (b) the link between them is more valuable than the links that have already been formed.

We now provide two examples of more general oligopoly models that satisfy the conditions of Proposition 4. For both examples, we assume that the cost function is given by equation 1 .

Example 4 Differentiated Cournot Oligopoly. Suppose each firm $i$ faces the following linear inverse demand function: $p_{i}=\alpha-q_{i}-\beta \sum_{j \neq i} q_{j}$, where $p_{i}$ is the price of the product sold by firm $i, \alpha>0$, and $\beta \in(0,1)$. In the Cournot equilibrium, the gross profit for firm $i$ is given by: $\Pi_{i}^{d}(g)=\theta\left(\mathcal{V}_{i}(g), \mathcal{V}\left(g_{-i}\right)\right)=\left(a_{1}+a_{2} \mathcal{V}_{i}(g)-a_{3} \mathcal{V}\left(g_{-i}\right)\right)^{2}$, with $a_{1}, a_{2}$, and $a_{3}$ as positive parameters. ${ }^{15}$ Moreover, to ensure that each firm produces a strictly positive quantity in equilibrium, assume that $a_{1}>a_{3} \mathcal{V}\left(g_{-i}\right)$ for all $\mathcal{V}\left(g_{-i}\right)$.

Example 5 Differentiated Bertrand Oligopoly. Suppose the demand function is similar to those given in Example 4. In the Bertrand equilibrium, the gross profit for firm $i$ is given by: $\Pi_{i}^{B}(\boldsymbol{g})=$ $\theta_{i}\left(\mathcal{V}_{i}(g), \mathcal{V}\left(g_{-i}\right)\right)=\lambda\left(a_{1}+a_{2} \mathcal{V}_{i}(g)-a_{3} \mathcal{V}\left(g_{-i}\right)\right)^{2}$, with $\lambda, a_{1}, a_{2}$, and $a_{3}$ as positive parameters.

\footnotetext{
${ }^{15}$ Due to their cumbersome nature, the values of the parameters for Examples 4 and 5 are given in Appendix D.
} 
It is worth noting that Example 3 was based on the fact that the profit function is convex in its first argument and sub-modular. It follows that when $\sigma$ satisfies these two properties, as in Examples 4 and 5, a policy designed to increase the value of the most valuable links may lead to equilibrium networks that do not have them, while equilibrium networks obtained without this policy may have these links in equilibrium. This suggests caution regarding public policy aimed at promoting R\&D collaboration.

To study efficient networks, we need an additional condition on $\sigma$ regarding the role played by the links of other firms in the profit function of each firm.

Definition 5 The gross profit function, $\sigma$, is strictly convex in its second argument if for all $x$, $v>0, \sigma(x, y+v)-\sigma(x, y)>\sigma(x, y)-\sigma(x, y-v)$ for all $y$.

It is worth noting that Examples 4 and 5 satisfy this convexity property. Moreover, for efficient networks we also need two simple additional monotonicity preserving type assumptions, the first one on the consumer surplus part (Property PCS), the second one on the social welfare (Property PW).

Property PCS: Let the marginal cost of firms $\ell$ and $\ell^{\prime}$ be lower than the marginal cost of firms $j$ and $j^{\prime}$ respectively. Suppose that first we decrease the marginal cost of $j$ and $j^{\prime}$ by $v$, then we decrease the marginal cost of $\ell$ and $\ell^{\prime}$ by $v$. Then, the change in the consumer surplus is higher in the second case than in the first case.

Note that Property PCS is satisfied by the demand functions presented in this paper, for cost function given by (1).

Property PW: If the marginal costs of two firms decrease without any cost of link formation, then the social welfare increases. 
Property PW implies that for a given network, it is always efficient to increase the value of the links formed by the firms.

The next proposition establishes that if (a) two firms $j$ and $j^{\prime}$ have higher flow degree than firms $i$ and $i^{\prime}$ respectively, and (b) the value of the link $j j^{\prime}$ is sufficiently high with regard to those of $i i^{\prime}$, then in an efficient network firms $j$ and $j^{\prime}$ are linked when firms $i$ and $i^{\prime}$ are linked.

Proposition 5 Let $g$ be a non-empty efficient network with $\mathcal{V}_{i}(g) \leq \mathcal{V}_{j}(g)$ and $\mathcal{V}_{i^{\prime}}(g) \leq \mathcal{V}_{j^{\prime}}(g)$. Suppose $\sigma$ is strictly convex in its first and second arguments, $\sigma$ is sub-modular, and the properties PCS and PW are satisfied. If there exists a link between firms $i$ and $i^{\prime}$ and $v_{i, i^{\prime}} \leq v_{j, j^{\prime}}$, then there exists a link between firms $j$ and $j^{\prime}$.

Note that if we set $j=i$, then we can obtain a result in line with Proposition 2.

Proposition 5 shows that two elements play a role in the existence of a link between two firms $i$ and $j$ in an efficient network: the first one is the flow of knowledge that accrues to these two firms due to their collaborations with other firms in the network, relative to the flow of knowledge that accrues to other linked firms; the second one is the value of the link between $i$ and $j$ relative to the value of the existing links.

\section{Concluding remarks}

This paper extends the work of Goyal and Joshi (2003) on collaborative R\&D network formation by assuming that cost reducing innovations between pairs of firms are heterogeneous. Clearly if every link takes a different value, the model has many degrees of freedom and can easily generate a rich set of results. Hence after providing a necessary condition to check for a pairwise equilibrium network or an efficient network when all links can have different values, we limit the set of values in a systematic way, and provide the architectures of pairwise equilibrium networks for two stylized 
frameworks, the I-O and H-L frameworks. Not surprisingly, we find that results of Goyal and Joshi (2003), Westbrock (2010) and Billand et al. (2015) as special cases, since all links have the same value in these models. We then provide results for a larger class of games like differentiated Cournot and Bertrand games.

An interesting result regarding public policy is that when this policy is used to increase the value of links having the highest value, this policy can lower welfare, given that some of the most valuable links may disapear in equilibrium. This result highlights the difference between our framework and a framework without links value heterogeneity, that is $v_{i, j}=1$ for all firms $i, j$, but with heterogeneous costs of forming links. ${ }^{16}$ Recall that the result in Example 2 uses the fact that when we make the most valuable links better some firms that have formed these links have strong incentives to form additional links, including less valuable ones. Consequently, if some firms add links, then other firms may remove some of their links, including the most valuable links. Hence, due to the substitution of less valuable links for some of the most valuable ones, public policy aimed at increasing the worth of the most valuable links may lead to welfare loss. Observe that links value heterogeneity is crucial for this type of non-monotonicity result. Moreover, this result cannot be obtained in a framework where the costs of forming links are heterogeneous. Indeed, in that case, firms that benefit from a policy subsidizing the cheapest existing links, do not have any incentive to form additional links, and the mechanism that leads to a welfare loss in the value heterogeneity framework cannot occur in the cost heterogeneity framework.

In our model, we have assumed that when two firms collaborate and innovate, each of them benefits in an identical manner from the innovation. In practice this may not be so. As a final point, we now illustrate what happens if we relax this assumption. Suppose that when the link $i j$ is added to network $g$, the marginal cost of firm $i$ is reduced by $\tau_{i}$ and the marginal cost of firm $j$ is reduced by $\tau_{j}$ where $\tau_{i} \neq \tau_{j}$. Obviously, if $\tau_{i}$ is sufficiently high relative to $\tau_{j}$, then firm $j$ will never accept to

\footnotetext{
${ }^{16}$ In the theoretical literature on network formation, several papers examine this type of heterogeneity (see for instance Galeotti, Goyal, and Kamphorst, 2005 ; Billand, Bravard and Sarangi, 2011, 2013).
} 
form the link with $i$. Indeed, link $i j$ improves the competitiveness of firm $i$ so much more than the competitiveness of firm $j$ that the latter firm will lower its profit by forming the link $i j$. Due to this mechanism, firms have an incentive to form links only with other firms that will not increase their competitiveness by a too substantial amount. Therefore, we can obtain a "tyranny of the weakest" situation, that is a situation in which the least able firms to take advantage of $R \& D$ collaboration form links with each other, leaving out the most able firms. A detailed examination of this issue is left for future research.

\section{References}

[1] P. Billand, C. Bravard, and S. Sarangi. Strict Nash networks and partner heterogeneity. International Journal of Game Theory, 40(3):515-525, August 2011.

[2] P. Billand, C. Bravard, and S. Sarangi. Modeling resource flow asymmetries using condensation networks. Social Choice and Welfare, 41(3):537-549, 2013.

[3] Billand P., Bravard C., Durieu J., Sarangi S. Efficient networks for a class of games with global spillovers. Journal of Mathematical Economics, 61: 203-210, 2015.

[4] R. Blundell, R. Griffith, and J. van Reenen. Market share, market value and innovation in a panel of british manufacturing firms. Review of Economic Studies, 66(3):529-554, 1999.

[5] U. Blum, C. Fuhrmeister, P. Marek, and M. Titze. R\&D collaborations and the role of proximity. Regional Studies, 51(12): 1761-1773, 2017

[6] A. Galeotti, S. Goyal, and J. Kamphorst. Network formation with heteregeneous players. Games and Economic Behavior, 54(2):353-372, 2005.

[7] B. Gomes-Casseres, J. Hagedoorn, and A. Jaffe. Do alliances promote knowledge flows? Journal of Financial Economics, 80:5-33, 2006. 
[8] S. Goyal and S. Joshi. Networks of collaboration in oligopoly. Games and Economic Behavior, 43(1):57-85, 2003.

[9] S. Goyal and S. Joshi. Unequal connections. International Journal of Game Theory, 34(3):319$349,2006$.

[10] S. Goyal and J.L Moraga-Gonzalez. R\&D networks. The RAND Journal of Economics, 32(4):686-707, 2001.

[11] S. Goyal, J.L. Moraga-Gonzalez, and A. Konovalov. Hybrid R\&D. Journal of the European Economic Association, 6(6):1309-1338, December 2008.

[12] J. Hagedoorn. Inter-firm R\&D partnerships: An overview of major trends and patterns since 1960. Research Policy, 31(4):477-492, 2002.

[13] M. D. König, S. Battiston, M. Napoletano, and F. Schweitzer. The efficiency and stability of R\&D networks. Games and Economic Behavior, 75(2):694-713, 2012.

[14] M. D. König, C. J. Tessone, and Y. Zenou. Nestedness in networks: A theoretical model and some applications. Theoretical Economics, 9(3):695-752, 2014.

[15] N.V.R. Mahadev, and U.N. Peled. Threshold graphs and related topics. North Holland, Amsterdam, 1995.

[16] D.C. Mowery, J.E. Oxley, B.S. Silverman. Technological overlap and interfirm cooperation: implications for the resource-based view of the firm. Research Policy, 27(5):507-523, 1998.

[17] D. Persitz. Core-periphery R\&D collaboration networks. Working paper, 2014.

[18] W.W. Powell, D.R. White, K.W. Koput, and J. Owen-Smith. Network dynamics and field evolution: The growth of interorganizational collaboration in the life sciences. American Journal of Economics and Sociology, 110(4):1132-1205, 2005. 
[19] M.V. Tomasello, M. Napoletano, A. Garas, F.Schweitzer. The rise and fall of R\&D networks. Industrial and Corporate Change, 2016.

[20] N. Vonortas, K. Okamura. Research Partners. International Journal of Technology Management, 46(3/4):280-306, 2009.

[21] N. Vonortas, K. Okamura. Network Structure and Robustness: Lessons for Research Programme Design. Economics of Innovation and New Technology, 46(3/4):280-306, 2013.

[22] N. Vonortas, L. Zirulia. Strategic Technology Alliances and Networks. Economics of Innovation and New Technology, 22(4):392-411, 2015.

[23] B. Westbrock. Natural concentration in industrial research collaboration. The RAND Journal of Economics, 41(2):351371, 2010.

\section{Appendix}

\section{Appendix A. Definitions}

Nested Split Graph (Mahadev and Peled, 1995) Let $g$ be a network whose distinct positive degrees are $\delta_{1}<\ldots<\delta_{m}$, and let $\delta_{0}=0$ (even if no firm of degree 0 exists). Let $D_{k}(g)=\left\{i \in N:\left|g_{i}\right|=\delta_{k}\right\}$ for $k \in\{0, \ldots m\}$. The sequence $D_{0}(g), \ldots, D_{m}(g)$ is called the degree partition of $G$. In a nested split graph (NSG) $g$, we have for each firm $i \in D_{\ell}(g), \ell \in\{1, \ldots, m\}$,

$$
g(i)= \begin{cases}\bigcup_{j=1}^{\ell} D_{m+1-j}(g), & \text { if } \ell=1, \ldots,\left\lfloor\frac{m}{2}\right\rfloor, \\ \bigcup_{j=1}^{\ell} D_{m+1-j}(g) \backslash\{i\}, & \text { if } \ell=\left\lfloor\frac{m}{2}\right\rfloor+1, \ldots, m .\end{cases}
$$




\section{Appendix B. Homogeneous Cournot Game}

In Appendix B and C, we let $\Delta_{1} \varphi\left(\mathcal{V}_{i}(g), \mathcal{V}\left(g_{-i}\right) ; v_{i j}\right)=\varphi\left(\mathcal{V}_{i}(g)+v_{i, j}, \mathcal{V}\left(g_{-i}\right)\right)-\varphi\left(\mathcal{V}_{i}(g), \mathcal{V}\left(g_{-i}\right)\right)$ and $\Delta_{2} \varphi\left(\mathcal{V}_{i}(g), \mathcal{V}\left(g_{-i}\right) ; v_{\ell, j}\right)=\varphi\left(\mathcal{V}_{i}(g), \mathcal{V}\left(g_{-i}\right)+v_{\ell, j}\right)-\varphi\left(\mathcal{V}_{i}(g), \mathcal{V}\left(g_{-i}\right)\right)$. Moreover, we let $S_{\varphi}(x, y, h)=$ $\frac{\varphi(x+h, y)-\varphi(x, y)}{h}$.

First, note that in the homogeneous Cournot game $\Delta_{1} \varphi$ is increasing in its first argument and decreasing in its second argument.

Proof of Proposition 1 First, note that since $\varphi(x, y)=(a+b x-c y)^{2}$, we have:

$$
S_{\varphi}(x, y ; h)=\bar{\varphi}_{1}^{\prime}(x, y ; h)
$$

Second, since $g$ is a pairwise equilibrium network where player $i$ and $j$ have formed a link, we have

$$
\Delta_{1} \varphi\left(\mathcal{V}_{i}(g)-v_{i j}, \mathcal{V}\left(g_{-i}\right) ; v_{i j}\right) \geq f
$$

We now prove successively the two parts of the proposition

1. Suppose that

$$
\frac{v}{v_{i, j}} \leq \frac{\bar{\varphi}_{1}^{\prime}\left(\mathcal{V}_{i}(g), \mathcal{V}\left(g_{-i}\right) ; v_{i, j}\right)}{\bar{\varphi}_{1}^{\prime}\left(\mathcal{V}_{i}(g)-v, \mathcal{V}\left(g_{-i}\right) ; v\right)}
$$

Then, we have

$$
\frac{v}{v_{i, j}} \leq \frac{S_{\varphi}\left(\mathcal{V}_{i}(g), \mathcal{V}_{-i}(g) ; v_{i, j}\right)}{S_{\varphi}\left(\mathcal{V}_{i}(g)-v, \mathcal{V}\left(g_{-i}\right) ; v\right)}
$$

and so $\Delta_{1} \varphi\left(\mathcal{V}_{i}(g), \mathcal{V}\left(g_{-i}\right) ; v_{i, j}\right) \geq \Delta_{1} \varphi\left(\mathcal{V}_{i}(g)-v, \mathcal{V}\left(g_{-i}\right) ; v\right)$. It follows that $\Delta_{1} \varphi\left(\mathcal{V}_{i}(g), \mathcal{V}\left(g_{-i}\right)\right.$; $\left.v_{i, j}\right) \geq f$, and firm $i$ has an incentive to form a link with firm $j$. By using similar arguments, we obtain that firm $j$ has an incentive to form a link with firm $i$. Consequently, $g$ is not a pairwise equilibrium, a contradiction.

2. We assume that firms $i, j \in N \backslash\left\{i^{\prime}, j^{\prime}\right\}$ are linked in $g$, and $v_{i^{\prime}, j^{\prime}} \geq v$. Moreover, suppose that $\mathcal{V}_{j^{\prime}}(g) \geq \mathcal{V}_{i^{\prime}}(g)$, and $\mathcal{V}_{i^{\prime}}(g)=\mathcal{V}_{i}(g)-v$, and $\bar{\varphi}_{1}^{\prime}\left(\mathcal{V}_{i^{\prime}}(g), \mathcal{V}\left(g_{-i^{\prime}}\right) ; v_{i^{\prime}, j^{\prime}}\right) \geq \bar{\varphi}_{1}^{\prime}\left(\mathcal{V}_{i^{\prime}}(g), \mathcal{V}\left(g_{-i^{\prime}}\right)-v ; v\right)$. 
To introduce a contradiction, we assume that $i^{\prime} j^{\prime} \notin g$. Since $\bar{\varphi}_{1}^{\prime}\left(\mathcal{V}_{i^{\prime}}(g), \mathcal{V}\left(g_{-i^{\prime}}\right) ; v_{i^{\prime}, j^{\prime}}\right) \geq$ $\bar{\varphi}_{1}^{\prime}\left(\mathcal{V}_{i^{\prime}}(g), \mathcal{V}\left(g_{-i^{\prime}}\right)-v ; v\right)$, we have: $S_{\varphi}\left(\mathcal{V}_{i^{\prime}}(g), \mathcal{V}\left(g_{-i^{\prime}}\right) ; v_{i^{\prime}, j^{\prime}}\right) \geq S_{\varphi}\left(\mathcal{V}_{i^{\prime}}(g), \mathcal{V}\left(g_{-i^{\prime}}\right)-v ; v\right)$. Therefore, we have $v S_{\varphi}\left(\mathcal{V}_{i^{\prime}}(g), \mathcal{V}\left(g_{-i^{\prime}}\right) ; v_{i^{\prime}, j^{\prime}}\right) \geq v S_{\varphi}\left(\mathcal{V}_{i^{\prime}}(g), \mathcal{V}\left(g_{-i^{\prime}}\right)-v ; v\right)$. Since $v_{i^{\prime}, j^{\prime}} \geq v$, we have $\left.\left.v_{i^{\prime}, j^{\prime}} S_{\varphi}\left(\mathcal{V}_{i^{\prime}}(g), \mathcal{V}\left(g_{-i^{\prime}}\right) ; v_{i^{\prime}, j^{\prime}}\right)\right) \geq v S_{\varphi}\left(\mathcal{V}_{i^{\prime}}(g), \mathcal{V}\left(g_{-i^{\prime}}\right) ; v_{i^{\prime}, j^{\prime}}\right)\right)$. Consequently, we have $\Delta_{1} \varphi\left(\mathcal{V}_{i^{\prime}}(g)\right.$, $\left.\mathcal{V}\left(g_{-i^{\prime}}\right) ; v_{i^{\prime}, j^{\prime}}\right) \geq \Delta_{1} \varphi\left(\mathcal{V}_{i^{\prime}}(g), \mathcal{V}\left(g_{-i^{\prime}}\right)-v ; v\right)=\Delta_{1} \varphi\left(\mathcal{V}_{i}(g)-v, \mathcal{V}\left(g_{-i}\right) ; v\right) \geq 0$. It follows that firm $i^{\prime}$ has an incentive to form a link with $j^{\prime}$ in $g$. By using similar arguments, we obtain that $j^{\prime}$ has an incentive to form a link with $i^{\prime}$ in $g$. Consequently, $g$ is not a pairwise equilibrium network, a contradiction.

Finally, by construction all the pairs in $\left(\mathcal{V}, v^{\prime} ; \mathcal{V}\left(g_{-i^{\prime}}\right)\right)_{\geq}$lead to marginal net profits higher or equal to $\Delta_{1} \varphi\left(\mathcal{V}, \mathcal{V}\left(g_{-i^{\prime}}\right) ; v^{\prime}\right)$. Since we have $\left(V_{i^{\prime}}(g), v_{i^{\prime}, j^{\prime}}\right)$ in $\left(\mathcal{V}, v^{\prime} ; \mathcal{V}\left(g_{-i^{\prime}}\right)\right)_{\geq}$, it follows that firm $i^{\prime}$ has an incentive to form a link with $j^{\prime}$. Since $\mathcal{V}_{j^{\prime}}(g) \geq \mathcal{V}_{i^{\prime}}(g)$ and $\mathcal{V}\left(g_{-j^{\prime}}\right) \leq \mathcal{V}\left(g_{-i^{\prime}}\right)$, we have $\Delta_{1} \varphi\left(\mathcal{V}_{j^{\prime}}(g), \mathcal{V}\left(g_{-j^{\prime}}\right) ; v_{i^{\prime}, j^{\prime}}\right) \geq \Delta_{1} \varphi\left(\mathcal{V}_{i^{\prime}}(g), \mathcal{V}\left(g_{-i^{\prime}}\right) ; v_{i^{\prime}, j^{\prime}}\right)$. Consequently, firms $i^{\prime}$ and $j^{\prime}$ have an incentive to form a link in $g$.

Proof of Corollary 1 We prove successively the two parts of the proposition.

1. Suppose that $v_{i, j} \geq v$. Then, we have

$$
\frac{\bar{\varphi}_{1}^{\prime}\left(\mathcal{V}_{i}(g), \mathcal{V}\left(g_{-i}\right) ; v_{i, j}\right)}{\bar{\varphi}_{1}^{\prime}\left(\mathcal{V}_{i}(g)-v, \mathcal{V}\left(g_{-i}\right) ; v\right)}=1+\frac{b\left(v_{i, j}+v\right)}{2\left(a+b \mathcal{V}_{i}(g)-c \mathcal{V}\left(g_{-i}\right)\right)-b v}>1 \geq \frac{v}{v_{i, j}}
$$

because $b>0$ and $2 a+2 b \mathcal{V}_{i}(g)-2 c \mathcal{V}\left(g_{-i}\right)-b v>0$. The result follows by Proposition 1.1.

2. Suppose that $\mathcal{V}_{j^{\prime}} \geq \mathcal{V}_{i^{\prime}} \geq \mathcal{V}_{i}$ and $v_{i^{\prime}, j^{\prime}} \geq v$. We are in special case of Proposition 1.2. We have

$$
\begin{aligned}
\Delta_{1} \varphi\left(\mathcal{V}_{i^{\prime}}(g), \mathcal{V}\left(g_{-i^{\prime}}\right) ; v_{i^{\prime}, j^{\prime}}\right) & =2 b v_{i^{\prime}, j^{\prime}}\left(a+b \mathcal{V}_{i^{\prime}}(g)-c \mathcal{V}\left(g_{-i^{\prime}}\right)\right)+b^{2} v_{i^{\prime}, j^{\prime}}^{2} \\
& \geq 2 b v\left(a+b \mathcal{V}_{i}(g)-c \mathcal{V}\left(g_{-i}\right)\right)-b^{2} v^{2} \\
& =\Delta_{1} \varphi\left(\mathcal{V}_{i}(g)-v, \mathcal{V}\left(g_{-i}\right) ; v\right) \\
& \geq f,
\end{aligned}
$$


because $\mathcal{V}_{i^{\prime}}(g) \geq \mathcal{V}_{i}(g), \mathcal{V}\left(g_{-i^{\prime}}\right) \leq \mathcal{V}\left(g_{-i}\right)$, and $v_{i^{\prime}, j^{\prime}} \geq v$

It follows that firm $i$ (resp. $j$ ) has an incentive to form a link with $j$ (resp. $i$ ). Consequently, $g$ is not a pairwise equilibrium network, a contradiction.

Proof of Corollary 2. Let $g$ be a non-empty pairwise stable network. First, we establish that $g$ is a group-dominant network, or a 2-group-dominant network, or a 2|2-hierarchical network, or a 1|2-hierarchical network. By Corollary 1.1, if firm $i \in N_{I O}^{k}, k \in\{1,2\}$, is involved in a link with firm $j \in N_{I O}^{k}$ in $g$, then $i$ has an incentive to form a link with all other firms in $N_{I O}^{k}$. Moreover, by Corollary 1.1 and the fact that $v^{I}>v^{O}$, if firm $i \in N_{I O}^{k}, k \in\{1,2\}$, is involved in a link with a firm $j \in N_{I O}^{-k}$, then $i$ has an incentive to form a link with all firms in $N$. It follows that $(i)$ two firms which belong to the same group and have formed links must be linked together, (ii) two firms that have formed inter-groups links must be linked together. Let us now establish successively the three parts of the proposition.

1. Let $g$ be a 2-group-dominant network. Moreover, suppose that $\left|N_{I O}^{1}(g)\right| \leq\left|N_{I O}^{2}(g)\right|$. We show that each component consists of firms which belong to the same group $N_{I O}^{k}$. To introduce a contradiction, suppose that there exists a link between firm $i \in N_{I O}^{1}$ and firm $j \in N_{I O}^{2}$. Since there are two components there exists firm $i^{\prime}$ which has formed a link in $g$ and which is not connected with $i$ and $j$. Wlog, we suppose that $i^{\prime} \in N_{I O}^{1}$. By Corollary 1.1, firms $i$ and $i^{\prime}$ have an incentive to form a link. Consequently, $g$ is not a pairwise equilibrium network, a contradiction.

2. Let $g$ be a 2|2-hierarchical network. First, note that if firm $i \in N_{I O}^{k}$ has formed a link with firm $j^{\prime} \in N_{I O}^{-k}$, then $i$ has an incentive to form a link with all firms in $N$ by Corollary 1.1. Moreover, by Corollary 1.1, if firm $i$ has formed a link with firm $j \in N_{I O}^{k}$, then $i$ has an incentive to form a link with all firms in $N_{I O}^{k}(g)$. 
3. Let $g$ be a $1 \mid 2$-hierarchical network where some firms in $N_{I O}^{k}(g)$ have formed links with all other firms in $N_{I O}^{-k}(g)$. We use the same type of arguments as in point 2.

Proof of Corollary 3. Let $g$ be a non-empty pairwise stable network. First, we establish that $g$ is a group-dominant network, or a 2-group-dominant network, or a 1|(1,1)-hierarchical network, or a 1|2-hierarchical network. Consider firm $i \in N^{H}$ that has formed a link with firm $j \in N^{L}$. By Corollary 1.1 and the fact that $v^{H}>v^{M}$, firm $i$ has an incentive to form a link with all firms in $N$. Consider firm $i \in N^{H}$ that has formed a link with $i^{\prime} \in N^{H}$ and has formed no links with firms in $N^{L}$. Then, by Corollary 1.1 firm $i$ has formed a link with all firms in $N^{H}(g)$. Consider firm $j \in N^{L}$ that has formed a link with $j^{\prime} \in N^{L}$. Then, by Corollary 1.1, firm $i$ has formed a link with every firm $j^{\prime} \in N^{L}(g)$. The result follows. Moreover, these arguments allow to prove the last part of the proposition. We now establish successively the two first parts of the corollary.

1. Let $g$ be a 2-group-dominant network. Then there are two components $C^{1}$ and $C^{2}$. Consider that component $C^{1}$ contains only firms $N^{H}$ and component $C^{2}$ contains only firms in $N^{L}$. To introduce a contradiction suppose that $C^{1}$ contains a firm $i \in N^{k}$ and $C^{2}$ contains $j \in N^{k}$, then by Corollary 1.1, $i$ and $j$ are linked in $g$. Let us now show that $\left|N^{H}(g)\right|<\left|N^{L}(g)\right|$. To introduce a contradiction suppose that $\left|N^{H}(g)\right| \geq\left|N^{L}(g)\right|$. We know that $v^{H}>v^{L}$. We have for firms $i \in N^{H}(g)$ and $i^{\prime} \in N^{L}(g), \mathcal{V}_{i}(g) \geq \mathcal{V}_{i^{\prime}}(g)$. By Corollary 1.1 and the fact that $v^{M}>v^{L}$, it follows that firm $i^{\prime}$ has an incentive to form a link with firm $i$. Moreover by Corollary 1.2 and the fact that $v^{M}>v^{L}$, firm $i$ has an incentive to form a link with firm $i^{\prime}$. It follows that $i$ and $i^{\prime}$ are linked in $g$, a contradiction.

2. Let $g$ be a 1|2-hierarchical network. Since firms in $N^{L}(g)$ have an incentive to form links with all firms, we obtain the result. We now establish that $\left|N^{H}(g) \backslash N^{H L}(g)\right|<N^{L}(g)$. To introduce a contradiction, suppose that $\left|N^{H}(g) \backslash N^{H L}(g)\right| \geq N^{L}(g)$. Then, for firms $i \in N^{H}(g) \backslash N^{H L}(g)$ and $j \in N^{L}(g)$, we have $\mathcal{V}_{i}(g)=\left(\left|N^{H}(g)\right|-1\right) v^{H}=\left(\left|N^{H}(g) \backslash N^{H L}(g)\right|-1\right) v^{H}+\left|N^{H L}(g)\right| v^{H}>$ 
$\left(\left|N^{L}(g)\right|-1\right) v^{L}+\left|N^{H L}(g)\right| v^{M}=\mathcal{V}_{j}(g)$. It follows that firm $i$ has an incentive to form a link with $j$, a contradiction.

\section{Appendix C. Welfare and Networks}

\section{Appendix C1.}

Proof of Proposition 2. Let $g$ be an efficient network which contains the link between $i$ and $j$. Let $v_{i, j}=v$ and $v_{i, j^{\prime}}=v^{\prime}$, with $v^{\prime} \geq v$. To introduce a contradiction, we suppose that $c_{j}(g) \geq c_{j^{\prime}}(g)$, i.e., $\mathcal{V}_{j}(g) \leq \mathcal{V}_{j^{\prime}}(g)$, and there is no link between $i$ and $j^{\prime}$. Consider network $g^{\prime}=g+i j^{\prime}$. In the following, we show that the incremental welfare associated with link $i j^{\prime}$ in $g$ is higher than the incremental welfare associated with link $i j$ in $g-i j$. It is sufficient to show that $W\left(g^{\prime}\right)-W(g)>W(g)-W(g-i j)$. The proof is divided into two steps. First, we establish that $W\left(g^{\prime}\right)-W(g)>W(g)-W(g-i j)$ where $v_{i j^{\prime}}=v_{i j}$. Second, let $g^{\prime \prime}$ be the network similar to $g^{\prime}$ except that $v^{\prime}=v_{i j^{\prime}}>v_{i j}=v$. We show that $W\left(g^{\prime \prime}\right)-W\left(g^{\prime}\right) \geq 0$.

1. We show that $W\left(g^{\prime}\right)-W(g)>W(g)-W(g-i j)$.

First, we establish that the total profit of the firms increases if the link between firms $i$ and $j^{\prime}$ is added.

We deal with firms $j$ and $j^{\prime}$. We show that $\Delta_{1} \varphi\left(\mathcal{V}_{j^{\prime}}(g), \mathcal{V}\left(g_{-j^{\prime}}\right) ; v\right)+\Delta_{2} \varphi\left(\mathcal{V}_{j}(g), \mathcal{V}\left(g_{-j}\right) ; v\right) \geq$ $\Delta_{1} \varphi\left(\mathcal{V}_{j}(g)-v, \mathcal{V}\left(g_{-j}\right) ; v\right)+\Delta_{2} \varphi\left(\mathcal{V}_{j^{\prime}}(g), \mathcal{V}\left(g_{-j^{\prime}}\right)-v ; v\right)$. Since $\mathcal{V}_{j^{\prime}}(g) \geq \mathcal{V}_{j}(g)$ and $\mathcal{V}\left(g_{-j}\right) \geq \mathcal{V}_{i}\left(g_{-j^{\prime}}\right)$ we have $\Delta_{1} \varphi\left(\mathcal{V}_{j^{\prime}}(g), \mathcal{V}\left(g_{-j^{\prime}}\right) ; v\right)-\Delta_{1} \varphi\left(\mathcal{V}_{j}(g)-v, \mathcal{V}\left(g_{-j}\right) ; v\right)=2 v b\left(b v+b\left(\mathcal{V}_{j^{\prime}}(g)-\mathcal{V}_{j}(g)\right)+c\left(\mathcal{V}\left(g_{-j}\right)-\right.\right.$ $\left.\mathcal{V}\left(g_{-j^{\prime}}\right)\right)>0$ and $\Delta_{2} \varphi\left(\mathcal{V}_{j}(g), \mathcal{V}\left(g_{-j}\right) ; v\right)-\Delta_{2} \varphi\left(\mathcal{V}_{j^{\prime}}(g), \mathcal{V}\left(g_{-j^{\prime}}\right)-v ; v\right)=2 c v\left(c v+c\left(\mathcal{V}\left(g_{-j}\right)-\mathcal{V}\left(g_{-j^{\prime}}\right)\right)+\right.$ $b\left(\mathcal{V}_{j^{\prime}}(g)-\mathcal{V}_{j}(g)\right)>0$. We now deal with firm $i$, we have $\Delta_{1} \varphi\left(\mathcal{V}_{i}(g), \mathcal{V}\left(g_{-i}\right) ; v\right)-\Delta_{1} \varphi\left(\mathcal{V}_{i}(g)-v, \mathcal{V}\left(g_{-i}\right) ;\right.$

$v)=2 b^{2} v^{2}$. Consequently, the incremental profits of firm $i, j$ and $j^{\prime}$ associated with the link $i j^{\prime}$ is higher than the incremental profits of firms $i, j$ and $j^{\prime}$ associated with the link $i j$. 
We now deal with firms in $N \backslash\left\{i, j, j^{\prime}\right\}$. For each firm $i^{\prime} \in N \backslash\left\{i, j, j^{\prime}\right\}, \Delta_{2} \varphi\left(\mathcal{V}_{i^{\prime}}(g), \mathcal{V}\left(g_{-i^{\prime}}\right) ; v\right)-$ $\Delta_{2} \varphi\left(\mathcal{V}_{i^{\prime}}(g), \mathcal{V}\left(g_{-i^{\prime}}\right)-v ; v\right)=2(c v)^{2}>0$. Therefore, the incremental loss incurred by $i^{\prime} \in N \backslash\left\{i, j, j^{\prime}\right\}$, when the link $i j^{\prime}$ is formed, is lower than the incremental loss incurred by $i^{\prime}$, when the link $i j$ is formed. To sum up, if the total profit increases when the link $i j$ is added, then the total profit also increases when the link $i j^{\prime}$ is added.

Second, we show that the incremental consumer surplus when the link $i j^{\prime}$ is added is higher than the incremental consumer surplus when the link $i j$ has been added. We have $\phi(\mathcal{V}(g)+v)-\phi(\mathcal{V}(g))-$ $(\phi(\mathcal{V}(g))-\phi(\mathcal{V}(g)-v))=(2 \gamma v /(n+1))^{2}>0$.

The result follows.

2. We now show that $W\left(g^{\prime \prime}\right)-W\left(g^{\prime}\right) \geq 0$. Let $v^{\prime \prime}=v^{\prime}-v$.

The addition of the costless link between firms $i$ and $j^{\prime}$ increases the profits of $i$ and $j^{\prime}$ by

$$
\left(\frac{\gamma v^{\prime \prime}(n-1)}{(n+1)^{2}}\right)\left(4\left(\alpha-\gamma_{0}\right)+2 \gamma(n-1) v^{\prime \prime}+2 \gamma(n-1)\left(\mathcal{V}_{i}+\mathcal{V}_{j^{\prime}}\right)-4 \gamma \sum_{j \in N \backslash\left\{i, j^{\prime}\right\}} \mathcal{V}_{j}\right)
$$

and decreases the profits of other firms by

$$
-\left(\frac{\gamma v^{\prime \prime}}{(n+1)^{2}}\right)\left(\left(4\left(\alpha-\gamma_{0}\right)-4 \gamma v^{\prime \prime}\right)(n-2)-4 \gamma(n-2)\left(\mathcal{V}_{i}+\mathcal{V}_{j^{\prime}}\right)+12 \gamma \sum_{j \in N \backslash\left\{i, j^{\prime}\right\}} \mathcal{V}_{j}\right)
$$

The addition of the link between firms $i$ and $j^{\prime}$ increases the consumer surplus by

$$
\frac{2 \gamma v^{\prime \prime}}{(n+1)^{2}}\left(n\left(\alpha-\gamma_{0}\right)+\gamma \sum_{i \in N} \mathcal{V}_{i}+\gamma v^{\prime \prime}\right)
$$

We have $A=(4(n-1)-4(n-2)+2 n)\left(\alpha-\gamma_{0}\right)=2(n+2)\left(\alpha-\gamma_{0}\right) \geq 4 \gamma(n+2)(n-1)^{2} \bar{v}$ by $(C 1)$. Moreover, we have $(-4(n-1)-12+2) \gamma \sum_{j \in N \backslash\left\{i, j^{\prime}\right\}} \mathcal{V}_{j}=-2 \gamma(2 n+3) \sum_{j \in N \backslash\left\{i, j^{\prime}\right\}} \mathcal{V}_{j} \geq$ $-2 \gamma(2 n+3)(n-2)(n-1) \bar{v}=-\mathcal{B}$. Since $4 \gamma(n+2)(n-1)^{2} \bar{v}>2 \gamma(2 n+3)(n-2)(n-1) \bar{v}$, we have $\mathcal{A}>\mathcal{B}$. 
Moreover, we have $2 \gamma\left((n-1)^{2}+2(n-2)+1\right)\left(\mathcal{V}_{i}(g)+\mathcal{V}_{j^{\prime}}\right) \geq 0$, and $2 \gamma\left((n-1)^{2}+2(n-2)+1\right) v^{\prime \prime} \geq 0$. The welfare increases if a costless link is added in the network.

It follows that $W\left(g^{\prime \prime}\right)-W(g) \geq W\left(g^{\prime}\right)-W(g)>W(g)-W(g-i j)$, and $g$ is not an efficient network, a contradiction.

Proof of Corollary 4. Let $g$ be an efficient network.

1. We show that if $i, j \in N^{t}, t \in\{1,2\}$, and $\mathcal{V}_{i}(g) \geq \mathcal{V}_{j}(g)$, then $g(i) \supseteq g(j)$. If $\mathcal{V}_{i}(g) \geq \mathcal{V}_{j}(g)$, then $c_{i}(g) \leq c_{j}(g)$. If $j^{\prime} \in g(j)$, then firm $j^{\prime}$ has formed a link with $j$ in $g$. By Proposition 2 since $c_{i}(g) \leq c_{j}(g)$ and $v_{j^{\prime}, j}=v_{j^{\prime}, i}=v$, then there is a link between firms $j^{\prime}$ and $i$ in $g$, that is $j^{\prime} \in g(i)$.

2. We show that if $i, j \in N^{t}, t \in\{1,2\}$, then we have either $g(i) \supseteq g(j)$ or $g(j) \supseteq g(i)$.

For all $i, j \in N^{t}$, we have $\mathcal{V}_{i}(g) \geq \mathcal{V}_{j}(g)$, or $\mathcal{V}_{j}(g) \geq \mathcal{V}_{i}(g)$. By 1 , in the first case we have $g(i) \supseteq g(j)$, and in the second case we have $g(j) \supseteq g(i)$.

3. We deal with sub-networks $g\left[N^{t}\right], t \in\{1,2\}$. Let a binary relation $\succeq$ on $N^{t}$ be defined as in (??). Then $\succeq$ is the vicinal preorder of $g\left[N^{t}\right], t \in\{1,2\}$. We consider the following morphism: $i \succeq j \Leftrightarrow \mathcal{V}_{i}(g) \geq \mathcal{V}_{j}(g)$. By point 2., the vicinal preorder, $\succeq$, of $g\left[N^{t}\right]$ is total. It follows that $g\left[N^{t}\right], t \in\{1,2\}$, is a NSG (see Appendix A).

4. We now show that a non-empty efficient network $g$ cannot be such that $g\left[N^{1}\right]=g\left[N^{2}\right]=\emptyset$. To introduce a contradiction, suppose a non-empty efficient network $g$ such that $g\left[N^{1}\right]=g\left[N^{2}\right]=$ $\emptyset$. Consider a firm $i \in N$ such that $|g(i)| \geq|g(j)|$, i.e., $\mathcal{V}_{i}(g) \geq \mathcal{V}_{j}(g)$ for every firm $j \in N$. Assume w.l.o.g. that $i \in N^{1}$. We distinguish between two cases. First, $i$ is the only firm in $N^{1}$ who has formed links. Consider a link $i j \in g$ with $j \in N^{2}$ and a firm $k \in N^{1}$. We build the network $g^{\prime}$ with $g^{\prime}=g-i j+i k$. Suppose that $v^{I}=v^{O}$. It is clear that $W\left(g^{\prime}\right)=W(g)$. Since $v^{I}>v^{O}$, by using the arguments given in the second part of the proof of Proposition 2, we have $W\left(g^{\prime}\right)>W(g)$ which contradicts the fact that $g$ is an efficient network. Second, consider 
a firm $k \in N^{1} \backslash\{i\}$ linked with a firm $j \in N^{2}$. Since $\mathcal{V}_{i}(g) \geq \mathcal{V}_{j}(g)$ and $v_{k i}=v^{I}>v^{O}=v_{k j}$, by Proposition 2, if the link $k j$ exists in an efficient network, then the link $k i$ exists. This contradicts the fact that $g$ is an efficient network.

5. We deal with the links between sub-networks $g\left[N^{t}\right], t \in\{1,2\}$. Let $\delta_{1}^{t}<\ldots<\delta_{m}^{t}$ be the distinct positive degrees of firms in $g\left[N^{t}\right], t \in\{1,2\}$, and let $\delta_{0}^{t}=0$ (even if no firm of degree 0 exists). let $D_{k}^{t}(g)=\left\{i \in N:\left|g_{i}\right|=\delta_{k}\right\}$ for $k \in\left\{0, \ldots m^{t}\right\}$. The sequence $\mathcal{D}^{t}=$ $D_{0}^{t}(g), \ldots, D_{m}^{t}(g)$ is called the degree partition of $g\left[N^{t}\right]$. Since $\left[i, j \in N^{t}, \mathcal{V}_{i}(g) \geq \mathcal{V}_{j}(g)\right] \Rightarrow$ $[g(i) \supseteq g(j)]$, if $i \in D_{k}^{t}$ and $j \in D_{k^{\prime}}^{t}$ in $g\left[N^{t}\right]$, with $k>k^{\prime}$, then $g(i) \cap\left(N \backslash N^{t}\right) \supseteq g(j) \cap\left(N \backslash N^{t}\right)$.

To sum up, suppose that $g\left[N^{1}\right] \neq \emptyset$ and $g\left[N^{2}\right] \neq \emptyset$. There are two possibilities: either there are no links between $g\left[N^{1}\right]$ and $g\left[N^{2}\right]$ and $g$ is a 2-NSG by point 3., or there are links between $g\left[N^{1}\right]$ and $g\left[N^{2}\right]$, and $g$ is a multi-NSG, by points 3. and 5. Suppose that $g\left[N^{t}\right] \neq \emptyset$ and $g\left[N \backslash N^{t}\right]=\emptyset$. Then by point 1., $g$ is a NSG or by point $5 . g$ is a group-NSG. Suppose that $g\left[N^{t}\right]=\emptyset$ and $g\left[N \backslash N^{t}\right]=\emptyset$, for $t=1$ or $t=2$. Then by point $4 ., g$ is empty.

The proof of Corollary 5 needs the following lemma.

Lemma 1 Let the assumptions of the H-L framework be satisfied. Suppose that network $g$ is such that $g\left[N^{L}\right]$ and $g\left[N^{H}\right]$ are non-empty NSGs and there are no link between these subnetworks. Consider a firm $i \in N^{L}$ with $k>0$ links and a firm $j \in N^{H}$. Let $g^{\prime}$ be similar to $g$ except that each link il $\in g$ is replaced by a link $j \ell$. Then $W\left(g^{\prime}\right)>W(g)$.

Proof We set $\lambda=\alpha-\gamma_{0}$. Let $\Lambda=W\left(g^{\prime}\right)-W(g)$ be the difference of welfare in networks $g$ and $g^{\prime}$ times $(n+1)^{2}$. We have

$$
\begin{aligned}
\Lambda= & \sum_{i \in N}\left(\lambda+n \gamma \mathcal{V}_{i}\left(g^{\prime}\right)-\gamma \sum_{j \in N \backslash\{i\}} \mathcal{V}_{j}\left(g^{\prime}\right)\right)^{2}+1 / 2\left(n \lambda+2 \gamma \mathcal{V}\left(g^{\prime}\right)\right)^{2} \\
& -\sum_{i \in N}\left(\lambda+n \gamma \mathcal{V}_{i}(g)-\gamma \sum_{j \in N \backslash\{i\}} \mathcal{V}_{j}(g)\right)^{2}+1 / 2(n \lambda+2 \gamma \mathcal{V}(g))^{2}
\end{aligned}
$$


Since the number of links is the same in $g$ and $g^{\prime}$, it is sufficient to show that $\Lambda>0$ to establish the lemma. We do this in two steps.

1. First, let $v^{M}=v^{L}$. In this case, we have

$$
\Lambda=2 \gamma^{2}(n+1)^{2} k v^{L} V_{j}(g) \geq 0 .
$$

2. Second, by using the same reasoning as in the proof of Proposition 2, point 2., we can conclude that $\Lambda \geq 0$ for $v^{M}=v^{L}$ implies $\Lambda>0$ for $v^{M}>v^{L}$.

Proof of Corollary 5. Let $g$ be an efficient network.

1. By using similar arguments as in the proof of Corollary 4, we establish that if $i, j \in N^{t}$, $t \in\{H, L\}$, and $\mathcal{V}_{i}(g) \geq \mathcal{V}_{j}(g)$, then $g(i) \supseteq g(j)$. It follows that if sub-network $g\left[N^{t}\right]$, $t \in\{H, L\}$ is non-empty, then it is a NSG.

2. We now establish that a 2-NSG cannot be an efficient network. To introduce a contradiction suppose that $g$ is a 2-NSG that is $g\left[N^{L}\right]$ and $g\left[N^{H}\right]$ are NSG and there is no link between firms in $N^{L}$ and firms in $N^{H}$. By construction, there exists a firm, say $i^{L} \in N^{L}$, such that $g\left(i^{L}\right) \neq \emptyset$. We now build the network $g^{\prime}$ similar to $g$ except that every firm $j^{L} \in g\left(i^{L}\right)$ replaces its link with $i^{L}$ by a link with $i^{H} \in N^{H}$. By Lemma $1, W\left(g^{\prime}\right)>W(g)$, a contradiction.

3. We now show that a non-empty efficient network $g$ cannot be such that $g\left[N^{H}\right]=g\left[N^{L}\right]=\emptyset$. To introduce a contradiction, suppose that $g\left[N^{H}\right]=g\left[N^{L}\right]=\emptyset$. First, suppose $g$ is such that for firm $i^{H} \in N^{H}$ we have $\left|g\left(i^{H}\right)\right| \geq\left|g\left(i^{L}\right)\right|>0$ for some $i^{L} \in N^{L}$. By applying the same arguments as in the proof of point 4 of Corollary 3, we obtain a contradiction. Second, if $g$ is such that for each $i^{H} \in N^{H}$ we have $\left|g\left(i^{H}\right)\right|<\left|g\left(i^{L}\right)\right|$ for all $i^{L} \in N^{L}$. Consider a network $g^{\prime}$ built as follows: each $i^{H} \in N^{H}$ becomes $i^{L} \in N^{L}$ and reciprocally. Observe that 
$W\left(g^{\prime}\right)=W(g)$. And $g^{\prime}$ is such that for a firm $i^{H} \in N^{H}$ we have $\left|g^{\prime}\left(i^{H}\right)\right| \geq\left|g^{\prime}\left(i^{L}\right)\right|>0$ for some $i^{L} \in N^{L}$. By applying the same arguments as in the previous case, the result follows.

4. We now show that if $g\left[N^{H}\right]=\emptyset$, then $g\left[N^{L}\right]=\emptyset$. To introduce a contradiction suppose that $g\left[N^{H}\right]=\emptyset$ and $g\left[N^{L}\right] \neq \emptyset$. By construction, there exists a firm, say $i^{L} \in N^{L}$, such that $g\left(i^{L}\right) \neq \emptyset$. By using the same argument as in point 2 , we obtain a contradiction.

To sum up, suppose that $g\left[N^{H}\right] \neq \emptyset$ and $g\left[N^{L}\right] \neq \emptyset$. By point 2., there are links between $g\left[N^{H}\right]$ and $g\left[N^{L}\right]$ and by 1., $g$ is a multi-NSG. Suppose that $g\left[N^{t}\right] \neq \emptyset$ and $g\left[N \backslash N^{t}\right]=\emptyset$. Then by point 4 ., $N^{t}=N^{H}$, and by point 1. $g$ is a NSG or a group-NSG. Suppose that $g\left[N^{t}\right]=\emptyset$ and $g\left[N \backslash N^{t}\right]=\emptyset$. Then by point $3 ., g$ is empty.

\section{Appendix C2.}

In the following, we will deal with situations where the number of links is given. Therefore, for the profits of firms, we need to deal only with the gross profit function. Let $A=\alpha-\gamma_{0}$, and let $\mathcal{V}=\left(\mathcal{V}_{1}, \mathcal{V}_{2}, \ldots, \mathcal{V}_{n}\right)$ be the vector of flow degrees of firms. Moreover, since the gross profit of a firm $i \in N$ depends on its flow degree, and the sum of the flow degrees of the other firms, for convenience, we denote the gross profit function of $i$ as $\hat{\Pi}_{i}\left(\mathcal{V}_{i}, \sum_{j \neq i} \mathcal{V}_{j}\right)$, and the Cournot equilibrium gross profit function of $i$ as $\hat{\varphi}_{i}\left(\mathcal{V}_{i}, \sum_{j \neq i} \mathcal{V}_{j}\right)$. Moreover, since the consumers surplus depends on the total flow degrees of firms, for convenience, we denote the consumers surplus function as $\widehat{C S}\left(\sum_{i \in N} \mathcal{V}_{i}\right)$. Finally, denote the social welfare function as $\hat{W}(\mathcal{V})=\hat{\Pi}_{i}\left(\mathcal{V}_{i}, \sum_{j \neq i} \mathcal{V}_{j}\right)+\widehat{C S}\left(\sum_{i \in N} \mathcal{V}_{i}\right)$

To establish Proposition 3, we need the two following lemmas.

Lemma 2 Let $\mathcal{V}$ and $\mathcal{V}^{\prime}$ be such that for two firms $i \in N$ and $j \in N$, and $t>0$, we have $\mathcal{V}_{i}^{\prime}=\mathcal{V}_{i}+t$, and $\mathcal{V}_{j}^{\prime}=V_{j}-t$, and for all firms $k \in N, k \neq i, j$, we have $\mathcal{V}_{k}^{\prime}=\mathcal{V}_{k}$. If $\mathcal{V}_{i}^{\prime} \geq \mathcal{V}_{j}$, then $\hat{W}\left(\mathcal{V}^{\prime}\right) \geq \hat{W}(\mathcal{V})$.

\section{Proof}


First, since $\sum_{i \in N} \mathcal{V}^{\prime}=\sum_{i \in N} \mathcal{V}$, we have $\widehat{C S}\left(\sum_{i \in N} \mathcal{V}_{i}^{\prime}\right)=\widehat{C S}\left(\sum_{i \in N} \mathcal{V}_{i}\right)$. Second, for each firm $k \neq i, j$, we have $\mathcal{V}_{k}^{\prime}=\mathcal{V}_{k}$, and $\sum_{j \neq k} \mathcal{V}_{j}^{\prime}=\sum_{j \neq k} \mathcal{V}_{j}$. It follows that $\hat{\varphi}_{k}\left(\mathcal{V}_{k}^{\prime}, \sum_{j \neq k} \mathcal{V}_{j}^{\prime}\right)=$ $\hat{\varphi}_{k}\left(\mathcal{V}_{k}, \sum_{j \neq k} \mathcal{V}_{j}\right)$

Third, if $\mathcal{V}_{i}^{\prime} \geq \mathcal{V}_{j}$, we have:

$\hat{\varphi}_{i}\left(\mathcal{V}_{i}^{\prime}, \sum_{\ell \neq i} \mathcal{V}_{\ell}^{\prime}\right)-\hat{\varphi}_{i}\left(\mathcal{V}_{i}, \sum_{\ell \neq i} \mathcal{V}_{\ell}\right)+\hat{\varphi}_{j}\left(\mathcal{V}_{j}^{\prime}, \sum_{\ell \neq j} \mathcal{V}_{\ell}^{\prime}\right)-\hat{\varphi}_{j}\left(\mathcal{V}_{j}, \sum_{\ell \neq j} \mathcal{V}_{\ell}\right)=2 \gamma^{2} t(n+1)^{2}\left(\mathcal{V}_{i}^{\prime}-\mathcal{V}_{j}\right) \geq 0$

The result follows

Lemma 3 Let $\mathcal{V}$ and $\mathcal{V}^{\prime}$ such that for a firm $i \in N$, we have $\mathcal{V}_{i}^{\prime}=\mathcal{V}_{i}+t$, and for all firms $k \in N, k \neq i$, we have $\mathcal{V}_{k}^{\prime}=\mathcal{V}_{k}$. Then, we have $\hat{W}\left(\mathcal{V}^{\prime}\right)>\hat{W}(\mathcal{V})$.

\section{Proof}

By computation, we have:

$$
\hat{W}\left(\mathcal{V}^{\prime}\right)-\hat{W}(\mathcal{V})=\frac{1}{2} \gamma t X
$$

where $X=2 \gamma\left(2 n^{2} \mathcal{V}_{i}+2 n \mathcal{V}_{i}-1\right)+\gamma t\left(2 n^{2}+2 n-1\right)+2(n+4) A-2 \gamma(2 n+3) \sum_{k \neq i} \mathcal{V}_{k}$.

By (C1), we have $A>2 \gamma(n-1)^{2} v^{I}$. Moreover, since for all $k \neq i$, we have $\mathcal{V}_{k}<(n-1) v^{I}$, it follows that we have $\sum_{k \neq i} \mathcal{V}_{k}<(n-1)^{2} v^{I}$. Then we have:

$$
X>2 \gamma\left(2 n^{2} \mathcal{V}_{i}+2 n \mathcal{V}_{i}-1\right)+\gamma t\left(2 n^{2}+2 n-1\right)+10 \gamma(n-1)^{2} v^{I}>0
$$

Since $X>0$, we have $\hat{W}\left(\mathcal{V}^{\prime}\right)-\hat{W}(\mathcal{V})=\frac{1}{2} \gamma t X>0$. The result follows.

\section{Proof of Proposition 3.}


Let $n^{k}=\left|N_{I O}^{k}\right|, k=1,2$, and $n^{k}(g)=\left|N_{I O}^{k}(g)\right|, k=1,2$. We write the set of the $n$ firms as $N=$ $\left\{i_{1}, i_{2}, \ldots, i_{n^{1}(g)}, i_{n^{1}(g)+1}, \ldots, i_{n^{1}}, i_{n^{1}+1}, \ldots, i_{n^{1}+n^{2}(g)}, i_{n^{1}+n^{2}(g)+1}, \ldots, i_{n}\right\}$, with $N^{1}=\left\{i_{1}, \ldots, i_{n^{1}}\right\}$, and $N^{2}=\left\{i_{n^{1}+1}, \ldots, i_{n}\right\}$. Denote by $\mathcal{V}(N)$ the vector of firms flow degrees in $g$ associated to the set $N$ : $\mathcal{V}(N)=\left(\mathcal{V}_{i_{1}}, \mathcal{V}_{i_{2}}, \ldots, \mathcal{V}_{i_{n^{1}}(g)}, \mathcal{V}_{i_{n^{1}(g)+1}}, \ldots, \mathcal{V}_{i_{n^{1}}}, \mathcal{V}_{i_{n^{1}+1}}, \ldots, \mathcal{V}_{i_{n^{1}+1+n^{2}(g)}}, \mathcal{V}_{i_{n^{2}(g)+1}}, \ldots, \mathcal{V}_{i_{n}}\right)$

Let the group-dominant networks $g$ and $g^{\prime}$ be such that $C(g)=\left\{i_{1}, \ldots, i_{n^{1}(g)}, i_{n^{1}+1}, \ldots, i_{n^{1}+n^{2}(g)}\right\}$. Set $i=i_{n^{1}(g)+1}$ and $j=i_{n^{1}+1}$. Hence, we have $C\left(g^{\prime}\right)=C(g) \cup\left\{i_{n^{1}(g)+1}\right\} \backslash\left\{i_{n^{1}+1}\right\}$.

To show that $W\left(g^{\prime}\right)>W(g)$, we use the following process.

Let $\mathcal{V}^{0}=\mathcal{V}(N)$. Let $\mathcal{V}^{t}$ be the vector of firms' flow degrees associated to the set of firms $N$ at Step $t$, and $\mathcal{V}_{i}^{t}$ be firm's $i$ flow degree at Step $t$. Note that in the following the vector $\mathcal{V}^{t}$ is only a sequence of flow degrees and is not particularly associated to a meaningful network in the I-O model.

Step 1. Define $\mathcal{V}^{1}$ as follows: $\mathcal{V}_{i_{n^{1}(g)+1}}^{1}=\mathcal{V}_{i_{n^{1}+1}}^{0}, \mathcal{V}_{i_{n^{1}+1}}^{1}=\mathcal{V}_{i_{n^{1}(g)+1}}^{0}$, and $\mathcal{V}_{k}^{1}=\mathcal{V}_{k}^{0}$ for all $k \neq$ $i_{n^{1}(g)+1}, i_{n^{1}+1}$. It is obvious that we have $\hat{W}\left(\mathcal{V}^{1}\right)=\hat{W}\left(\mathcal{V}^{0}\right)$.

Step 2. Define $\mathcal{V}_{i_{n^{1}(g)+1}}^{2}=\mathcal{V}_{i_{n^{1}(g)+1}}^{1}+\left(n^{1}(g)-n^{2}(g)+1\right)\left(v^{I}-v^{O}\right)$, and $\mathcal{V}_{k}^{2}=\mathcal{V}_{k}^{1}$ for all $k \neq i_{n^{1}(g)+1}$. Since $\left(n^{1}(g)-n^{2}(g)+1\right)\left(v^{I}-v^{O}\right)>0$, by Lemma 3 , we have $\hat{W}\left(\mathcal{V}^{2}\right)>\hat{W}\left(\mathcal{V}^{1}\right)$.

If $n^{2}(g)=1$, then we go to Step 4. Otherwise, we go to Step 3 .

Step 3. Fix $\ell=1$. Define $\mathcal{V}_{i_{\ell}}^{\ell+2}=\mathcal{V}_{i_{\ell}}^{\ell+1}+\left(v^{I}-v^{O}\right), \mathcal{V}_{i_{n^{1}+1+\ell}+2}=\mathcal{V}_{i_{n^{1}+1+\ell}+1}-\left(v^{I}-v^{O}\right)$, and $V_{k}^{\ell+2}=V_{k}^{\ell+1}$ for all $k \neq i_{\ell}, i_{n^{1}+1+\ell}$

We can check that $\mathcal{V}_{i_{\ell}}^{\ell+2} \geq \mathcal{V}_{i_{n^{1}+1+\ell}^{\ell+1}}$. Therefore, by Lemma 2 , we have $\hat{W}\left(\mathcal{V}^{\ell+2}\right) \geq \hat{W}\left(\mathcal{V}^{\ell+1}\right)$.

We repeat Step 3 for $\ell:=\ell+1$ until $\ell=n^{2}(g)$.

Step 4. Fix $\ell=n^{2}(g)$. Define $\mathcal{V}_{i_{\ell}}^{\ell+2}=\mathcal{V}_{i_{\ell}}^{\ell+1}+\left(v^{I}-v^{O}\right)$, and $V_{k}^{\ell+2}=V_{k}^{\ell+1}$ for all $k \neq i_{\ell}$. 
Since $v^{I}-v^{O}>0$, by Lemma 3 , we have $\hat{W}\left(\mathcal{V}^{\ell+2}\right)>\hat{W}\left(\mathcal{V}^{\ell+1}\right)$.

We repeat Step 4 for $\ell:=\ell+1$ until $\ell=n^{1}(g)+1$.

We can check that the vector $\mathcal{V}^{n^{1}(g)+2}$ is associated to the network $g^{\prime}$. The result follows.

Proof of Corollary 6. Let $n^{k}, n^{k}(g), N, \mathcal{V}(N)$, and $C(g)$ be defined as in the proof of Proposition 3. Let $C\left(g^{\prime}\right)$ be the dominant group in $g^{\prime}$ and $C\left(g^{\ell}\right)$ be the dominant group in group-dominant network $g^{\ell}$.

To show that $W\left(g^{\prime}\right)>W(g)$, we construct a sequence of group-dominant networks that allows to transit from the dominant group $C(g)$ to the dominant group $C\left(g^{\prime}\right)$. We show that each transition from one group-dominant network to the following in this sequence induces an increase of the social welfare.

Let $C\left(g^{0}\right)$ be the first dominant group in the sequence. We set $C\left(g^{0}\right)=C(g)$. Subsequently, each dominant group in the sequence is defined from the previous one as follows. Fix $\ell=1$. Define $C\left(g^{\ell}\right)=C\left(g^{\ell-1}\right) \backslash\left\{i_{n_{1}+\ell}\right\} \cup\left\{i_{n_{1}(g)+\ell}\right\}$. We repeat this construction for $\ell:=\ell+1$ until $\ell=n^{2}(g)+1$.

Observe that the number of links remains constant throughout the sequence. By Proposition 3, we know that $W\left(g^{\ell}\right)>W\left(g^{\ell-1}\right)$. And, by construction, $C\left(g^{n^{2}(g)}\right)$ corresponds to $C\left(g^{\prime}\right)$.

\section{Appendix D. Results for a Large Class of Oligopoly Games}

Proof of Proposition 4. First, we note that $v_{i, j}>0$ otherwise firm $i$ has formed a costly link that does not increase its gross profit. Second, to introduce a contradiction assume a pairwise equilibrium network $g$ that does not satisfy the condition given in the proposition. Hence, $\mathcal{V}_{j}(g) \leq \mathcal{V}_{j^{\prime}}(g)$ and 
$v_{i, j} \leq v_{i, j^{\prime}}$. Since $\mathcal{V}_{j}(g) \leq \mathcal{V}_{j^{\prime}}(g)$, we have $\mathcal{V}\left(g_{-j}\right) \geq \mathcal{V}\left(g_{-j^{\prime}}\right)$. So,

$$
\begin{aligned}
\Pi_{j^{\prime}}\left(g+i j^{\prime}\right)-\Pi_{j^{\prime}}(g) & =\Delta_{1} \sigma\left(\mathcal{V}_{j^{\prime}}(g), \mathcal{V}\left(g_{-j^{\prime}}\right) ; v_{i j^{\prime}}\right) \geq \Delta_{1} \sigma\left(\mathcal{V}_{j^{\prime}}(g), \mathcal{V}\left(g_{-j}\right) ; v_{i j^{\prime}}\right) \\
& \geq \Delta_{1} \sigma\left(\mathcal{V}_{j}(g), \mathcal{V}\left(g_{-j}\right) ; v_{i j^{\prime}}\right) \geq \Delta_{1} \sigma\left(\mathcal{V}_{j}(g), \mathcal{V}\left(g_{-j}\right) ; v_{i j}\right) \\
& >\Delta_{1} \sigma\left(\mathcal{V}_{j}(g)-v_{i j}, \mathcal{V}\left(g_{-j}\right) ; v_{i j}\right)=\Pi_{i}(g)-\Pi_{i}(g-i j)
\end{aligned}
$$

The first inequality comes from the fact that $\mathcal{V}\left(g_{-j}\right) \geq \mathcal{V}\left(g_{-j^{\prime}}\right)$ and $\sigma$ is sub-modular. The second inequality comes from the fact that $\mathcal{V}_{j}(g) \leq \mathcal{V}_{j^{\prime}}(g)$ and $\sigma$ is strictly convex in its first argument. The third inequality comes from the fact that $v_{i j^{\prime}} \geq v_{i j}$ and $\sigma$ is strictly increasing in its first argument. The strict inequality comes from the fact that $v_{i j}>0$ and $\sigma$ is strictly convex in its first argument. For firm $i$, by using similar argument as above we have $\Delta_{1} \sigma\left(\mathcal{V}_{i}(g), \mathcal{V}\left(g_{-i}\right) ; v_{i j^{\prime}}\right) \geq \Delta_{1} \sigma\left(\mathcal{V}_{i}(g)\right.$, $\left.\mathcal{V}\left(g_{-i}\right) ; v_{i j}\right)>\Delta_{1} \sigma\left(\mathcal{V}_{i}(g)-v_{i, j}, \mathcal{V}\left(g_{-i}\right) ; v_{i j}\right)$. Since $\Pi_{i}(g)-\Pi_{i}(g-i j) \geq f$ and $\Pi_{j}(g)-\Pi_{j}(g-i j) \geq f$, firms $i$ and $j^{\prime}$ have an incentive to form a link together. Therefore, network $g$ is not a pairwise equilibrium network, a contradiction.

Values of the parameters of Example 4 (Differentiated Cournot Oligopoly). The equilibrium gross profit for firm $i \in N$ is given by $\Pi_{i}^{d}(g)=\theta\left(\mathcal{V}_{i}(g), \mathcal{V}\left(g_{-i}\right)\right)=\left(a_{1}+a_{2} \mathcal{V}_{i}(g)-a_{3} \mathcal{V}\left(g_{-i}\right)\right)^{2}$, where

$$
\begin{aligned}
& a_{1}=\left(\alpha-\gamma_{0}\right) /(2+\beta(n-1)), \\
& a_{2}=\gamma((n-2) \beta+2) /((2-\beta)(2+\beta(n-1)), \\
& a_{3}=\gamma \beta /((2-\beta)(2+\beta(n-1)) .
\end{aligned}
$$

Values of the parameters of Example 5 (Differentiated Bertrand Oligopoly). The equilibrium gross profit for firm $i \in N$ can be written as: $\Pi_{i}^{B}(\boldsymbol{g})=\theta_{i}\left(\mathcal{V}_{i}(g), \mathcal{V}\left(g_{-i}\right)\right)=\lambda\left(a_{1}+a_{2} \mathcal{V}_{i}(g)-\right.$ 
$\left.a_{3} \mathcal{V}\left(g_{-i}\right)\right)^{2}$, where

$$
\begin{aligned}
\lambda & =\frac{(1-\beta)(1+(n-1) \beta)}{1+(n-2) \beta}, \\
a_{1} & =\frac{(1+(n-2 \beta))}{(2+(n-3) \beta)(1+(n-1) \beta)}\left(\alpha-\gamma_{0}\right), \\
a_{2} & =\gamma \frac{2+(5 n-11) \beta+\left(4 n^{2}-19 n+21\right) \beta^{2}+\left(\left(n^{2}-8 n+19\right) n-14\right) \beta^{3}}{(2+(n-3) \beta)(1+(n-1) \beta)(1-\beta)(2+(2 n-3) \beta)} \\
a_{3} & =2 \gamma \frac{\beta+(2 n-4) \beta^{2}+\left(n^{2}-4 n+4\right) \beta^{3}}{(2+(n-3) \beta)(1+(n-1) \beta)(1-\beta)(2+(2 n-3) \beta)} .
\end{aligned}
$$

To prove Proposition 5 we need the following lemma.

Lemma 4 Let $g$ be a network that maximizes the total profit, where $\mathcal{V}_{i}(g) \leq \mathcal{V}_{j}(g)$ and $\mathcal{V}_{i^{\prime}}(g) \leq$ $\mathcal{V}_{j^{\prime}}(g)$. If there exists a link between $i$ and $i^{\prime}$ in $g$, and $v_{i, i^{\prime}}=v_{j, j^{\prime}}$, then $j j^{\prime} \in g$.

Proof The method of proof used here is borrowed from Billand et al. (Proposition 1, 2015). Let $g$ be a network that maximizes the total profit. To introduce a contradiction suppose that $\mathcal{V}_{i}(g) \leq \mathcal{V}_{j}(g), \mathcal{V}_{i^{\prime}}(g) \leq \mathcal{V}_{j^{\prime}}(g), v_{i, i^{\prime}}=v_{j, j^{\prime}}=v$ and there is no link between $j$ and $j^{\prime}$. First, we establish that if the link between firms $j$ and $j^{\prime}$ is added, then the total profit of the firms increases. Note that $\mathcal{V}\left(g_{-i}\right) \geq \mathcal{V}\left(g_{-j}\right)$. We have

$$
\Delta_{1} \sigma\left(\mathcal{V}_{j}(g), \mathcal{V}\left(g_{-j}\right) ; v\right) \geq \Delta_{1} \sigma\left(\mathcal{V}_{j}(g), \mathcal{V}\left(g_{-i}\right) ; v\right)>\Delta_{1} \sigma\left(\mathcal{V}_{i}\left(g-i i^{\prime}\right), \mathcal{V}\left(g_{-i}\right) ; v\right)
$$

The first inequality follows the fact that $\sigma$ is sub-modular and the second inequality follows the fact that $\sigma$ is strictly convex in its first argument. It follows that the impact of the link $i i^{\prime}$ on the profit of firm $i$ is lower than the impact of the link between $j j^{\prime}$ on the profit of firm $j$. Using similar arguments will give us this result is true for firms $i^{\prime}$ and $j^{\prime}$.

Moreover, we have

$$
\left.\Delta_{2} \sigma\left(\mathcal{V}_{i}(g), \mathcal{V}\left(g_{-i}\right) ; v\right) \geq \Delta_{2} \sigma\left(\mathcal{V}_{j}(g), \mathcal{V}\left(g_{-i}\right) ; v\right) \geq \Delta_{2} \sigma\left(\mathcal{V}_{j}(g), \mathcal{V}\left(g_{-j}\right) ; v\right)\right)
$$


The first inequality follows the fact that $\sigma$ is sub-modular. Second inequality follows the fact that $\sigma$ is strictly convex in its second argument.

It follows that the loss of firm $i$ associated with $j j^{\prime}$ is lower than the loss of $j$ associated with $i i^{\prime}$. Using similar arguments, it can be easily shown that this result is true for firms $i^{\prime}$ and $j^{\prime}$.

Moreover, for each firm $\ell \in N \backslash\left\{i, i^{\prime}, j, j^{\prime}\right\}$, by the strict convexity in the second argument of $\sigma$ we have $\left.\Delta_{2} \sigma\left(\mathcal{V}_{\ell}(g), \mathcal{V}\left(g_{-\ell}\right) ; v\right)\right) \geq \Delta_{2} \sigma\left(\mathcal{V}_{\ell}(g), \mathcal{V}\left(g_{-\ell}\right)-v ; v\right)$. Therefore, the incremental loss incurred by $\ell$, when the link $j j^{\prime}$ is formed, is lower than the incremental loss incurred by $\ell$, when the link $i i^{\prime}$ is formed.

Let $\pi^{t}(g)$ the total profit function given $g$. Since $g$ maximizes the total profit, we have $\pi(g)-$ $\pi\left(g-i i^{\prime}\right) \geq 0$. We have established that $\pi\left(g+j^{\prime} j^{\prime}\right)-\pi(g)>\pi(g)-\pi\left(g-i i^{\prime}\right)$. It follows that $\pi\left(g+j^{\prime} j^{\prime}\right)-\pi(g)>0$, a contradiction.

Proof of Proposition 5. Let $\mathbf{V}(g)=\left(\mathcal{V}_{i_{1}}(g), \ldots, \mathcal{V}_{i_{\ell}}(g), \ldots, \mathcal{V}_{i_{n}}(g)\right)$, with $\mathcal{V}_{i_{\ell}}(g) \geq \mathcal{V}_{i_{\ell+1}}(g)$; and let $\mathbf{V}\left(g ; v_{i_{\ell}, i_{\ell^{\prime}}}\right)$ be the $n$-uple identical to $\mathbf{V}(g)$ except that $\mathcal{V}_{i_{\ell}}(g)$ and $\mathcal{V}_{i_{\ell}^{\prime}}(g)$ are replaced by $\mathcal{V}_{i_{\ell}}(g)+v_{i_{\ell}, i_{\ell^{\prime}}}$ and $\mathcal{V}_{i_{\ell}^{\prime}}(g)+v_{i_{\ell}, i_{\ell^{\prime}}}$ respectively. Similarly, let $\mathbf{V}\left(g ;-v_{i_{\ell}, i_{\ell^{\prime}}}\right)$ be the $n$-tuple identical to $\mathbf{V}(g)$ except that $\mathcal{V}_{i_{\ell}}(g)$ and $\mathcal{V}_{i_{\ell}^{\prime}}(g)$ are replaced by $\mathcal{V}_{i_{\ell}}(g)-v_{i_{\ell}, i_{\ell^{\prime}}}$ and $\mathcal{V}_{i_{\ell}^{\prime}}(g)-v_{i_{\ell}, i_{\ell^{\prime}}}$ respectively. We denote by $C S(g)=\Psi(\mathbf{V}(g))$ the consumer surplus function associated with $g$ and $\pi^{T}(\mathbf{V}(g),|g|)=$ $\sum_{i \in N} \sigma\left(\mathcal{V}_{i}(g), \mathcal{V}\left(g_{-i}\right)\right)$ the total profit function. The welfare function is given by: $W(\mathbf{V}(g),|g|)=$ $\Psi(\mathbf{V}(g))+\pi^{T}(\mathbf{V}(g),|g|)$.

We now write Properties PCS and PW in a formal form.

Property PCS: If $\ell>j, \ell^{\prime}>j^{\prime}, v_{i_{\ell}, i_{\ell^{\prime}}}=v_{i_{j}, i_{j^{\prime}}}=v$, then $\Psi\left(\mathbf{V}\left(g ; v_{i_{\ell}, i_{\ell^{\prime}}}\right)\right)-\Psi(\mathbf{V}(g))>$ $\Psi(\mathbf{V}(g))-\Psi\left(\mathbf{V}\left(g ;-v_{i_{j}, i_{j^{\prime}}}\right)\right)$.

Property PW: For $v_{i, j}>0, W\left(\mathbf{V}\left(g ; v_{i, j}\right),|g|\right)>W(\mathbf{V}(g),|g|)$.

Let $g$ be a network, with $i i^{\prime} \in g$ and $j j^{\prime} \notin g$. To introduce a contradiction suppose that $g$ is efficient. We assume that $\mathcal{V}_{i}(g) \leq \mathcal{V}_{j}(g), \mathcal{V}_{i^{\prime}}(g) \leq \mathcal{V}_{j^{\prime}}(g)$ 
1. Suppose $v_{i, i^{\prime}}=v_{j, j^{\prime}}=v$. By Lemma 4, given in Appendix D, we know that network $g^{\prime}$ that maximizes the total profit contains a link between firms $j$ and $j^{\prime}$ since it contains a link between firms $i$ and $i^{\prime}$. Moreover, since the assumptions of Property PCS are satisfied, network $g^{\prime}$ that maximizes the total consumer surplus contains a link between firms $j$ and $j^{\prime}$ since it contains a link between firms $i$ and $i^{\prime}$. It follows that if $v_{i, i^{\prime}}=v_{j, j^{\prime}}=v$, then $W\left(\mathbf{V}\left(g ; v_{j, j^{\prime}}\right),|g|+1\right)>W(\mathbf{V}(g),|g|)$, a contradiction.

2. Suppose $v_{j, j^{\prime}}>v_{i, i^{\prime}}$. Let $v_{j, j^{\prime}}^{\prime}=v_{j, j^{\prime}}-v_{i, i^{\prime}}>0$. By the previous point we have $W\left(\mathbf{V}\left(g ; v_{j, j^{\prime}}-\right.\right.$ $\left.v_{j, j^{\prime}}^{\prime},|g|+1\right)>W(\mathbf{V}(g),|g|)$, and by property PW, we have $W\left(\mathbf{V}\left(g ; v_{j, j^{\prime}},|g|+1\right)>W\left(\mathbf{V}\left(g ; v_{j, j^{\prime}}-\right.\right.\right.$ $\left.v_{j, j^{\prime}}^{\prime},|g|+1\right)$, a contradiction. 